\title{
Single-Cell Imaging of Bioenergetic Responses to Neuronal Excitotoxicity and Oxygen and Glucose Deprivation
}

\author{
(1) Niamh M.C. Connolly, ${ }^{1,2}$ Heiko Düssmann, ${ }^{1,2,3}$ Ujval Anilkumar, ${ }^{1,3}{ }^{-1}$ Heinrich J. Huber, ${ }^{1,2,4}$ and Jochen H.M. Prehn ${ }^{1,2,3}$ \\ ${ }^{1}$ Department of Physiology and Medical Physics, ${ }^{2}$ Centre of Systems Medicine, and ${ }^{3}$ Centre for the Study of Neurological Disorders, Royal College of \\ Surgeons in Ireland, Dublin 2, Ireland, and ${ }^{4}$ Department of Cardiovascular Sciences, KU Leuven, 3000 Leuven, Belgium
}

\begin{abstract}
Excitotoxicity is a condition occurring during cerebral ischemia, seizures, and chronic neurodegeneration. It is characterized by overactivation of glutamate receptors, leading to excessive $\mathrm{Ca}^{2+} / \mathrm{Na}^{+}$influx into neurons, energetic stress, and subsequent neuronal injury. We and others have previously investigated neuronal populations to study how bioenergetic parameters determine neuronal injury; however, such experiments are often confounded by population-based heterogeneity and the contribution of effects of non-neuronal cells. Hence, we here characterized bioenergetics during transient excitotoxicity in rat and mouse primary neurons at the single-cell level using fluorescent sensors for intracellular glucose, ATP, and activation of the energy sensor AMP-activated protein kinase (AMPK). We identified ATP depletion and recovery to energetic homeostasis, along with AMPK activation, as surprisingly rapid and plastic responses in two excitotoxic injury paradigms. We observed rapid recovery of neuronal ATP levels also in the absence of extracellular glucose, or when glycolytic ATP production was inhibited, but found mitochondria to be critical for fast and complete energetic recovery. Using an injury model of oxygen and glucose deprivation, we identified a similarly rapid bioenergetics response, yet with incomplete ATP recovery and decreased AMPK activity. Interestingly, excitotoxicity also induced an accumulation of intracellular glucose, providing an additional source of energy during and after excitotoxicity-induced energy depletion. We identified this to originate from extracellular, AMPKdependent glucose uptake and from intracellular glucose mobilization. Surprisingly, cells recovering their elevated glucose levels faster to baseline survived longer, indicating that the plasticity of neurons to adapt to bioenergetic challenges is a key indicator of neuronal viability.
\end{abstract}

Key words: bioenergetics; excitotoxicity; single-cell imaging

\section{Introduction}

Neuronal excitotoxicity is a condition caused by excessive release of the excitatory neurotransmitter glutamate that contributes to the pathophysiology of neurodegenerative disorders, ischemic stroke, and traumatic brain injury (Lee et al., 1999; Wang and Qin, 2010; Hardingham and Bading, 2010; Rodriguez-Rodriguez et al., 2013). Excess extracellular glutamate activates NMDA, AMPA, and kainic acid subtypes of glutamate receptors, leading to a disproportional calcium and sodium influx into neurons. Attempts to restore ion homeostasis result in an overactivation of ATP-dependent ion pumps, causing ATP depletion (Ankarcrona et al., 1995; Marcaida et al., 1995; Nicholls and Budd, 2000; Ward et al., 2007; Weisová et al., 2009; Concannon et al., 2010). We and

Received July 23, 2013; revised June 3, 2014; accepted June 8, 2014.

Author contributions: N.M.C.C., H.J.H., and J.H.M.P. designed research; N.M.C.C., H.D., and U.A. performed research; N.M.C.C., H.D., U.A., H.J.H., and J.H.M.P. analyzed data; N.M.C.C., H.J.H., and J.H.M.P. wrote the paper.

This research was supported by Grant 08/IN.1/B1949 from Science Foundation Ireland to H.J.H. and J.H.M.P., N.M.C.C. was the recipient of a postgraduate scholar fellowship funded by the Health Research Board in Ireland (PhD/2007/11). We thank Drs. Lewis C. Cantley, Wolf B. Frommer, and Hiroyuki Noji for plasmids used in this study. The authors declare no competing financial interests.

Correspondence should be addressed to either of the following: Dr. Jochen H.M. Prehn, Department of Physiology and Medical Physics, Royal College of Surgeons in Ireland, 123 Street, Stephen's Green, Dublin 2, Ireland, E-mail: prehn@rcsi.ie; or Dr. Heinrich J. Huber, Centre for Systems Medicine, Department of Physiology and Medical Physics, Royal College of Surgeons in Ireland, York House, York Street, Dublin 2, Ireland, E-mail: heinhuber@rcsi.ie.

DOI:10.1523/JNEUROSCI.3127-13.2014

Copyright $\odot 2014$ the authors $\quad 0270-6474 / 14 / 3410192-14 \$ 15.00 / 0$ others have previously shown that whether or not neurons survive or undergo apoptotic or necrotic cell death largely depends on the bioenergetic balance driven largely by the duration and severity of the insult (Ankarcrona et al., 1995; Bonfoco et al., 1995; Lipton and Nicotera, 1998; Ward et al., 2007; Weisová et al., 2009; Concannon et al., 2010). We also identified a cytoprotective mechanism during excitotoxic injury where the AMPactivated protein kinase (AMPK) was found to mediate the surface expression of the neuronal glucose transporter 3 (GLUT3), leading to increased glucose uptake and facilitating ATP recovery (Weisová et al., 2009).

The identification of novel molecular targets for cytoprotective intervention during excitotoxic injury may benefit from a more detailed investigation of the temporal alterations in bioenergetic parameters and their dependence on cell signaling. At the moment, knowledge of bioenergetic parameters during excitotoxicity, such as glucose and ATP levels or AMPK activity, derives primarily from studies performed in isolated mitochondria or in neuronal populations (Ankarcrona et al., 1995; Marcaida et al., 1995; Kushnareva et al., 2005; Ward et al., 2007; Weisová et al., 2009; Hattori et al., 2010). However, measured dynamics in cell populations can be slower than in individual cells due to unsynchronized responses, so that key events are often "averaged down" or may remain undetectable and cannot be directly correlated to individual cell outcome (Loewer and Lahav, 2011). 
Likewise, population-based measurements in neuronal cultures often include confounding factors such as contributions from dying neurons or from non-neuronal cells.

To address this deficiency, we used recently developed Förster resonance energy transfer (FRET)-based fluorescent reporters to detect alterations in glucose and ATP concentration, and in AMPK activity at the single-cell level during and after excitotoxicity and oxygen glucose deprivation (OGD)-induced neuronal injury. We show that ATP depletion and recovery during and after excitotoxicity is rapid and shows significant bioenergetic plasticity. We also demonstrate that excitotoxicity resulted in an accumulation of intracellular glucose, and that the ability to rapidly restore intracellular glucose homeostasis after excitotoxicity is a predictor of neuronal survival.

\section{Materials and Methods}

Materials. Fetal bovine serum (FBS), minimum essential medium (MEM; plus Earle's L-glutamine, Invitrogen), Fluo-4 AM, and tetramethylrhodamine methyl ester (TMRM) were from Invitrogen (BioScience). 5-Amino-imidazole-4-carboxamide $1 \beta$-D-ribofuranoside (AICAR) was from Cell Signaling Technology, while Compound C was from Merck Biosciences. Glutamate, NMDA, glycine, MK-801, and all other reagents were obtained from Sigma-Aldrich, unless otherwise stated. The following media and buffers were prepared: feeding media: cerebellar granule neurons (CGNs; MEM, $32 \mathrm{~mm}$ glucose, $18 \mathrm{~mm} \mathrm{KCl,} \mathrm{10 \%} \mathrm{FBS,} \mathrm{and} 200$ $\mathrm{U} / \mathrm{ml} / 200 \mu \mathrm{g} / \mathrm{ml}$ penicillin/streptomycin; cortical neurons (Corticals): MEM, $32 \mathrm{~mm}$ glucose, $0.5 \mathrm{~mm}$ L-glutamine, 5\% FBS, 5\% horse serum, and $200 \mathrm{U} / \mathrm{ml} / 200 \mu \mathrm{g} / \mathrm{ml}$ pen/strep; plating media: CGNs: feeding media 2 mM L-glutamine; Corticals: neurobasal medium embryonic, 2\% B27, $0.5 \mathrm{~mm}$ L-Glutamine, $200 \mathrm{U} / \mathrm{ml} / 200 \mu \mathrm{g} / \mathrm{ml}$ pen/strep; control buffer: 120 $\mathrm{mm} \mathrm{NaCl}, 3.5 \mathrm{~mm} \mathrm{KCl}, 0.4 \mathrm{~mm} \mathrm{KH}_{2} \mathrm{PO}_{4}, 5 \mathrm{~mm} \mathrm{NaHCO} 3$ (Riedel de Haën), $20 \mathrm{~mm}$ HEPES, pH 7.4, with $1.5 \mathrm{~mm} \mathrm{CaCl}_{2}$, and $15 \mathrm{~mm}$ glucose added just before the experiments. Whenever indicated, glucose was either omitted or substituted with $2 \mathrm{~mm}$ sodium pyruvate.

Preparation of rat cerebellar granule neurons and mouse cortical neurons. Cerebellar granule neurons were prepared from 7-day-old SpragueDawley rat pups of either sex and cortical neurons were prepared from embryonic day 16-18 mouse pups of either sex, as previously described (Ward et al., 2000; D'Orsi et al., 2012), under licenses granted by the Irish Department for Health and Children and with ethics approval from the Royal College of Surgeons in Ireland. Briefly, cerebella or cortices isolated from at least three pups were stored in ice-cold dissection buffer (PBS, $14 \mathrm{~mm}$ glucose, $45 \mu \mathrm{M}$ bovine serum albumin), and trypsinized $\left(0.25 \%, 25 \mathrm{~min}, 37^{\circ} \mathrm{C}\right)$. The pooled cell solution was centrifuged, resuspended in plating medium and strained ( $40 \mu \mathrm{m}$ pores; BD Falcon, BD). Cells were seeded at $\sim 2 \times 10^{5}$ cells $/ \mathrm{cm}^{2}$ on prewashed, poly-D-lysine ( 5 $\mu \mathrm{g} / \mathrm{ml}$ )-coated glass Will-Co dishes (WillCo Wells BV) or 24- and 6-well plates (Sarstedt), and incubated at $37^{\circ} \mathrm{C}$ with $5 \% \mathrm{CO}_{2}$. Twenty-four hours after seeding, cells were washed to remove dead cells, and media were replaced with 1:1 plating/feeding media supplemented with cytosine arabinose-C to inhibit non-neuronal cell proliferation $(10 \mu \mathrm{M}$ CGNs, 600 nм Corticals). Cortical neuron media was replaced with complete feeding media after $3 \mathrm{~d}$ in vitro (DIV). Cells were used after 7-10 DIV.

Transient neuronal transfection. Cells were transfected with fluorescent protein fusion plasmids designed for FRET measurements. CGNs were transfected after 5-7 DIV via a calcium-phosphate precipitate method (Dudek et al., 2001). Briefly, $25 \mathrm{~mm}$ N,N-bis(2-hydroxyethyl)-2aminoethansulfonic acid-buffered saline, $140 \mathrm{~mm} \mathrm{NaCl}$, and $0.75 \mathrm{~mm}$ $\mathrm{Na}_{2} \mathrm{HPO}_{4}$ was added to $1.75 \mu \mathrm{g}$ of DNA and $125 \mathrm{mM} \mathrm{CaCl}_{2}$. Transfection medium (MEM, 15 mm HEPES, 1 mm sodium pyruvate; Gibco), $1 \times$ glutamax (Gibco), 0.6\% glucose, $1 \times$ B27 (Gibco), pH 7.65, and the DNA mix were added to cells in a ratio of $6.25: 1$. Cells were incubated in a $\mathrm{CO}_{2}$-free incubator at $37^{\circ} \mathrm{C}$ for $1-2 \mathrm{~h}$ to allow sufficient precipitate formation ("sandy" appearance). Cells were washed three times in wash buffer (control buffer plus $1.2 \mathrm{~mm} \mathrm{MgCl}_{2}$ ). Conditioned media was replaced, and cells were returned to $5 \% \mathrm{CO}_{2}, 37^{\circ} \mathrm{C}$ incubation. Cortical neurons were transfected after 5-6 DIV using Lipofectamine 2000 reagent in Opti-MEM Medium (Invitrogen) according to the manufacturer's instructions. Cells were used $24-48 \mathrm{~h}$ after transfection.

Neuronal injury models-excitotoxicity and OGD. Glutamate/glycine or NMDA/glycine $(100 / 10 \mu \mathrm{M})$ was added on stage to CGNs or Corticals, respectively. NMDA receptor activation was ended after 5 or $10 \mathrm{~min}$ by the addition of $10 \mu \mathrm{M}$ MK-801 (Fluka), an NMDA receptor antagonist. Pharmacological compounds [2 $\mu \mathrm{g} / \mathrm{ml}$ oligomycin, $12 \mathrm{~mm}$ 2-deoxyglucose (2DG), $10 \mu \mathrm{M}$ compound C (CompC), $100 \mu \mathrm{M}$ 1,4-dideoxy-1,4-imino-Darabinitol hydrochloride (DAB), $100 \mu \mathrm{M}$ 1-deoxynojirimycin hydrochloride (NJM)] were added to the buffer directly on stage. Excitotoxicity experiments were performed in $15 \mathrm{~mm}$ extracellular glucose, unless explicitly stated.

For observation of single-cell kinetics during OGD, cells were places in a heated stage incubator allowing adjustment of $\mathrm{CO}_{2}$ and $\mathrm{O}_{2}$ concentrations (PeCon). To simulate OGD, media were replaced with ischemic CSF (ICSF; $70 \mathrm{~mm} \mathrm{NaCl}, 70 \mathrm{~mm} \mathrm{KCl}, 1.25 \mathrm{~mm} \mathrm{NaH}_{2} \mathrm{PO}_{4}, 2 \mathrm{~mm} \mathrm{MgSO}_{4}$, $0.3 \mathrm{~mm} \mathrm{CaCl}, 10 \mathrm{~mm}$ sucrose, $5.25 \mathrm{~mm} \mathrm{NaHCO}, 10 \mathrm{~nm}$ TMRM; bubbled with $\mathrm{N}_{2}$ ), and $\mathrm{O}_{2}$ levels were reduced to $1.5 \%$. After 40-45 min, ICSF media were replaced with conditioned media, and normoxic $\left(20 \% \mathrm{O}_{2}\right)$ conditions were restored. During some experiments, focus had to be readjusted after media exchange- out of focus images were not included in the analysis of single-cell kinetics, resulting in gaps in single-cell traces.

Determination of neuronal injury. Following exposure to NMDA, glutamate, or OGD, neurons were allowed to recover in conditioned media at $37^{\circ} \mathrm{C}, 5 \% \mathrm{CO}_{2}$ for $24 \mathrm{~h}$ before being stained live with Hoechst 33258 stain $(1 \mu \mathrm{g} / \mathrm{ml})$. The extent of neuronal injury compared with sham conditions (control buffer alone) was determined by the percentage of condensed pyknotic nuclei, as described previously (Concannon et al., 2010).

Fluorescence microscopy. The membrane-permeant, cationic fluorescent probe TMRM was used as a measure of changes in the mitochondrial membrane potential. All cells were equilibrated with TMRM (10 $\mathrm{nM}$ ) in control buffer at least $45 \mathrm{~min}$ before imaging (for OGD, cells were maintained in regular media before OGD). For measurements of intracellular calcium, cells were preincubated with Fluo-4 AM $(3 \mu \mathrm{M})$ for 45 min. Mineral oil was added on top of the buffer to minimize evaporation.

The ATeam 1.03' FRET-based probe, kindly provided by Dr. Hiroyuki Noji (Osaka University, Osaka, Japan), is a highly specific and sensitive reporter of cytosolic ATP concentration, and is $\mathrm{pH}$ insensitive around physiological $\mathrm{pH}$ (Imamura et al., 2009). ATP binding to the $\varepsilon$-subunit of the bacterial $F_{0} / F_{1}$-ATP synthase alters the probe conformation, increasing FRET (see Fig. 2A). The FRET-based AMPK activity reporter (AMPKAR), kindly provided by Dr. Lewis C. Cantley (Harvard Medical School, Boston, MA), contains a synthetic peptide incorporating an AMPK substrate motif. Specific AMPK phosphorylation of the threonine residue on the synthetic peptide induces a conformational change in the probe that increases FRET, as shown in Figure $4 A$ (Tsou et al., 2011). The probe is therefore an indicator of AMPK activity. The FLII ${ }^{12}$ Pglu$700 \mu \delta 6$ "glucose-FRET" probe (plasmid \#17866, Addgene) is a specific reporter of intracellular glucose concentration with a linear response range between 0.05 and $9.6 \mathrm{~mm}$ (Takanaga et al., 2008). Glucose binding to the glucose-galactose-binding protein alters the probe conformation and increases FRET (see Fig. 5A). This plasmid has been optimized to reduce $\mathrm{pH}$ sensitivity [enhanced yellow fluorescent protein (YFP) replaced with citrine protein] and other potential sources of artifact (Fehr et al., 2003; Takanaga et al., 2008). A control-FRET reporter (FLII ${ }^{12}$ PgluD183A; glucose-FRET probe with a one-residue mutation to reduce glucose sensitivity; Takanaga et al., 2008), was used as a negative control for glucose sensitivity, and was kindly provided by Dr. Wolf B. Frommer (Carnegie Institute, Stanford, CA). For interexperimental comparison, we normalized all FRET-based glucose measurements (15 and $0 \mathrm{~mm}$ extracellular glucose) to an average of the baseline ratiometric fluorescence signal measured in neurons incubated in $0 \mathrm{~mm}$ glucose (plus $2 \mathrm{~mm}$ pyruvate). As further negative controls to exclude potential artifacts affecting the fluorescent proteins, we used a FRET-based plasmid with a caspase-cleavable linker sequence [SCAT3 (enhanced cyan fluorescent protein (eCFP)-DEVD-Venus); Takemoto et al., 2003]. The SCAT3 ratiometric fluorescence signal remained unaltered during excitotoxic 
stimulation, which is in line with the reported absence of significant executioner caspase activity in models of excitotoxicity (Lankiewicz et al., 2000; Ward et al., 2006).

Glass bottom WillCo-dishes were mounted on a heated stage above a $40 \times$ or $63 \times$ oil-immersion objective lens. Inverted confocal laserscanning microscopes (LSM 510 Meta, 5live duoscan, and 710, Zeiss) were used with optimized polychroic mirror and filter wheel settings. Fluorophores were excited using a $405 \mathrm{~nm}$ (CFP) or $489 \mathrm{~nm}$ diode laser (YFP, Fluo-4), a $488 \mathrm{~nm}$ argon laser (YFP, Fluo-4), a $543 \mathrm{~nm}$ helium/ neon laser, or a $561 \mathrm{~nm}$ DPSS laser (TMRM). Emitted fluorescence was detected through bandpass or long-pass filters for the LSM 510 and 5live duoscan, according to the maximum emission wavelengths of the fluorophores (Fluo-4, $\sim 515 \mathrm{~nm}$; TMRM, $\sim 576 \mathrm{~nm}$; CFP, $\sim 475 \mathrm{~nm}$; YFP, $\sim 527 \mathrm{~nm}$ ). In the case of the LSM 710, the spectral detection bands were selected accordingly. Cells were imaged on stage for at least $10 \mathrm{~min}$ before treatment to measure baseline fluorescence. Images were obtained approximately every $1 \mathrm{~min}$ before, during, and for at least $10 \mathrm{~min}$ after treatment, and at $5 \mathrm{~min}$ intervals for longer experiments. Cells were imaged on stage for up to $16 \mathrm{~h}$ after treatment. Efforts were made to reduce phototoxicity by minimizing exposure time and laser excitation power.

Image processing. Images were processed using MetaMorph 7.564 bit (Molecular Devices). After background subtraction, YFP channel images were used to segment transfected cells. The ratiometric values shown in the traces were obtained by calculating the ratio image of the FRET/ CFP $\times 1000$ for the segmented cells, obtaining the kinetics of the mean value for each cell. The fluorescence signal referred to as FRET here is the fluorescent signal emitted in the YFP band after $405 \mathrm{~nm}$ excitation. For fluorescent dyes (TMRM and Fluo-4 AM), individual cells were isolated within regions of interest (ROIs), and the average signal intensity within the ROI was plotted over time. All traces shown are normalized to baseline (average of the first eight images) and are representative of at least three independent experiments, unless explicitly stated.

Intracellular glucose measurement by glucose oxidase assay. After 8 DIV, media in 6-well plates were replaced with control buffer. After $1 \mathrm{~h}$, Corticals/CGNs were treated with NMDA/glutamate for 5/10 min and allowed to recover for up to $2 \mathrm{~h}$. Neurons were washed three times in control buffer (containing no glucose) and lysed in hypotonic lysis buffer (100 mM Tris, $2.5 \mathrm{~mm}$ EDTA, $\mathrm{pH}$ 7.75) before being stored at $-80^{\circ} \mathrm{C}$. Intracellular glucose levels were measured using the Amplex Red Glucose Oxidase Assay kit (Invitrogen), following the manufacturer's instructions. Glucose oxidase reacts with glucose to produce gluconolactone and $\mathrm{H}_{2} \mathrm{O}_{2}$. The subsequent reaction of $\mathrm{H}_{2} \mathrm{O}_{2}$ with the Amplex Red Reagent in the presence of horseradish peroxidase generates a red fluorescent oxidation product whose fluorescence emission was measured at $595 \mathrm{~nm}$ after excitation at $560 \mathrm{~nm}$.

Statistical analysis. Single-cell data (time-stamp, fluorescence intensity value) were input to MATLAB R2007B (MathWorks) where trace characteristics (time to depolarization, area under the curve, and duration of recovery to baseline) were either manually identified or automatically calculated. In each box-and-whisker plot, the box has lines at the lower, median, and upper quartile values. The whiskers extend to the most extreme values within 1.5 times the interquartile range. Cells lying outside this range are indicated by filled circles. Mean \pm SEM values are shown in the bar charts.

Each experiment was performed on neurons from at least three independent preparations. To analyze single-cell behavior, each neuron's response was treated as an independent event in statistical analyses. The occurrence of significant differences between the medians was determined by Kruskal-Wallis test (followed by a multiple-comparison test) and rank sum/paired rank sum tests using Bonferroni correction for multiple pairwise comparisons. Differences in the means were calculated using the Student's $t$ test. Pearson and Spearman correlation tests were performed using the MATLAB function "corr." A $p$ value of $<0.05$ was considered to be significant $\left({ }^{*} p<0.05\right.$; $\left.{ }^{* *} p<0.01\right)$. The Kaplan-Meier curve, which reports the percentage of surviving neurons over time (each step in the curve represents one depolarization event, lowering the percentage), was generated in StataIC 12 (StataCorp). For survival analysis, surviving neurons from experiments that were performed for $<6 \mathrm{~h}$ were removed.

\section{Results}

Transient excitotoxic and OGD injury models induce changes in intracellular calcium and mitochondrial membrane potential

To investigate neuronal NMDA receptor-mediated glutamate excitotoxicity (Castilho et al., 1998; Ward et al., 2000), we transiently exposed rat CGNs (7-9 DIV) to glutamate and its coagonist glycine $(100 / 10 \mu \mathrm{M})$ for $10 \mathrm{~min}$ (Fig. $1 \mathrm{~A})$, and observed significantly increased cell death after $24 \mathrm{~h}$, compared with sham controls (Fig. 1B). We performed time-lapse confocal microscopy to monitor single CGNs before, during, and after the excitotoxic insult. Using Fluo-4 AM, a fluorescent reporter of intracellular calcium $\left(\mathrm{Ca}^{2+}\right)$, and TMRM, a cationic fluorescent dye reporting changes in mitochondrial membrane potential $\left(\Delta \Psi_{\mathrm{m}}\right)$, we confirmed a rapid and transient increase in intracellular $\mathrm{Ca}^{2+}$ and depletion in $\Delta \Psi_{\mathrm{m}}$ (Fig. 1C), which is consistent with previous results (Nicholls et al., 2003; Ward et al., 2007). This was followed in some neurons by delayed apoptotic death, characterized by delayed calcium deregulation and depolarization of $\Delta \Psi_{\mathrm{m}}$ (Fig. $1 C$, dotted trace). A second model of neuronal NMDA receptor-mediated excitotoxicity, where mouse Corticals were exposed to NMDA/glycine $(100 / 10 \mu \mathrm{M})$ for $5 \mathrm{~min}$ (Fig. 1D), exhibited similar $\mathrm{Ca}^{2+}$ and $\Delta \Psi_{\mathrm{m}}$ dynamics (D'Orsi et al., 2012) and also increased neuronal death after $24 \mathrm{~h}$ (Fig. 1E).

In addition, we extended our investigation to OGD, a widely used in vitro model of ischemic stroke, consisting of both excitotoxic and non-excitotoxic features (Goldberg and Choi, 1993). Implementing an oxygen regulation system that allowed us to continuously monitor single neurons within our confocal microscopy setting, we found that exposure of cortical neurons to OGD for $45 \mathrm{~min}$ (Fig. $1 F$ ) significantly increased cell death after $24 \mathrm{~h}$ (Fig. 1G). Similar to the observations in the pure excitotoxicity models above, we found that $\Delta \Psi_{\mathrm{m}}$ was transiently depleted during treatment, and recovered after restoration of normoxic and normoglycemic conditions (Fig. $1 H$ ). Interestingly, and in contrast to the excitotoxicity models, an initial increase in $\mathrm{Ca}^{2+}$ was quickly re-equilibrated during treatment, while a secondary increase in $\mathrm{Ca}^{2+}$ was measured after reoxygenation, with a more gradual return to baseline.

\section{ATP depletion during transient excitotoxicity is a rapid, transient process, and ATP recovery is incomplete after OGD.} We previously demonstrated that the above transient glutamate exposure depleted ATP levels in CGN populations, with complete ATP recovery within 1-2 h (Ward et al., 2007; Weisová et al., 2009). To investigate ATP dynamics in our single-cell system, we transiently transfected neurons with the recently developed FRET-based reporter of cytosolic ATP levels, ATeam (Imamura et al., 2009; Fig. 2A). We first confirmed that this tool was capable of reporting ATP levels in our system, by inhibiting glycolytic and mitochondrial ATP production (with 2-DG and oligomycin, respectively). As expected, the ATeam fluorescence signal collapsed only in the presence of both inhibitors (Fig. 2B; we observed similar behavior for oligomycin addition followed by 2-DG; data not shown), suggesting that either glycolytic or mitochondrial ATP production is sufficient to maintain ATP levels in CGNs at rest. Oligomycin addition in the presence of $0 \mathrm{~mm}$ extracellular glucose (where mitochondrial ATP production is assumed to be the only form of ATP production) also induced an immediate collapse of the ATeam fluorescence signal (data not shown). 
A

DIV 7-9 Cerebellar Granule Neurons
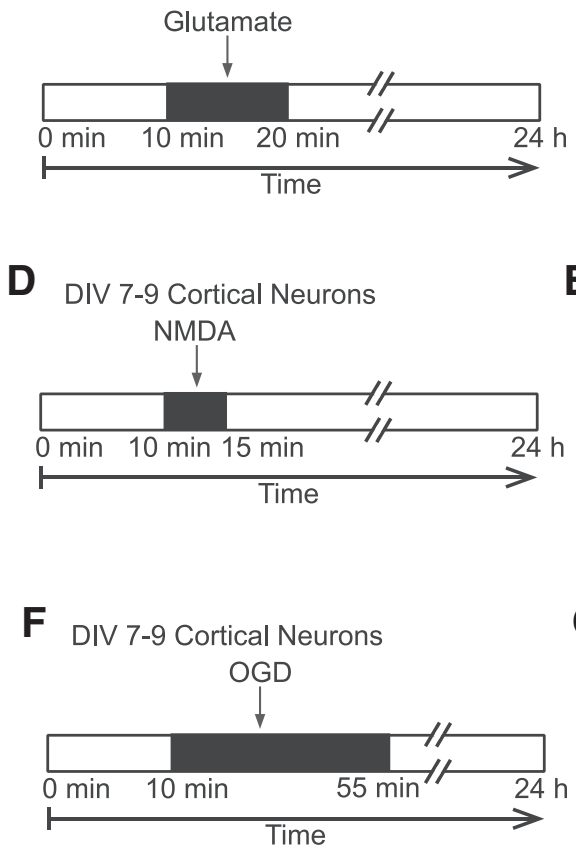

B
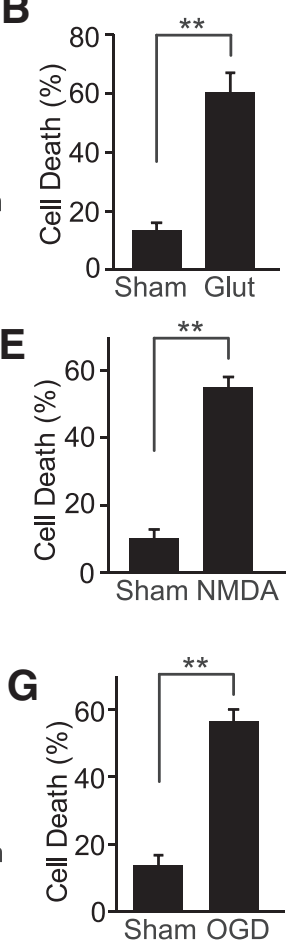

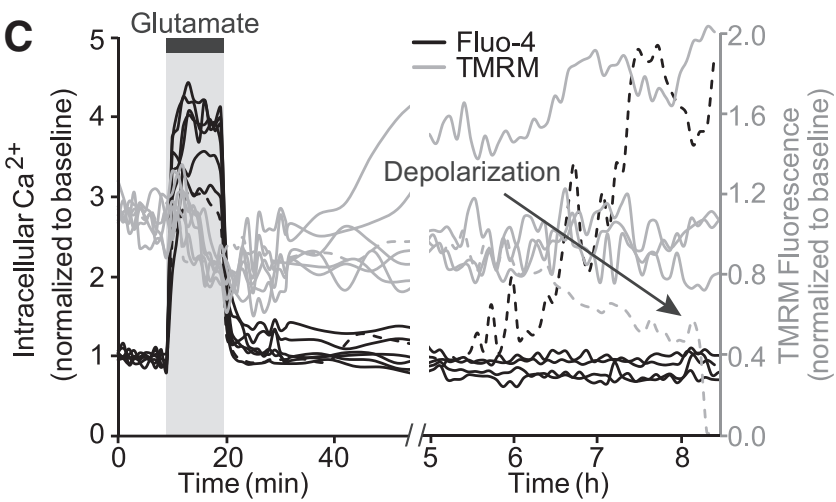

H

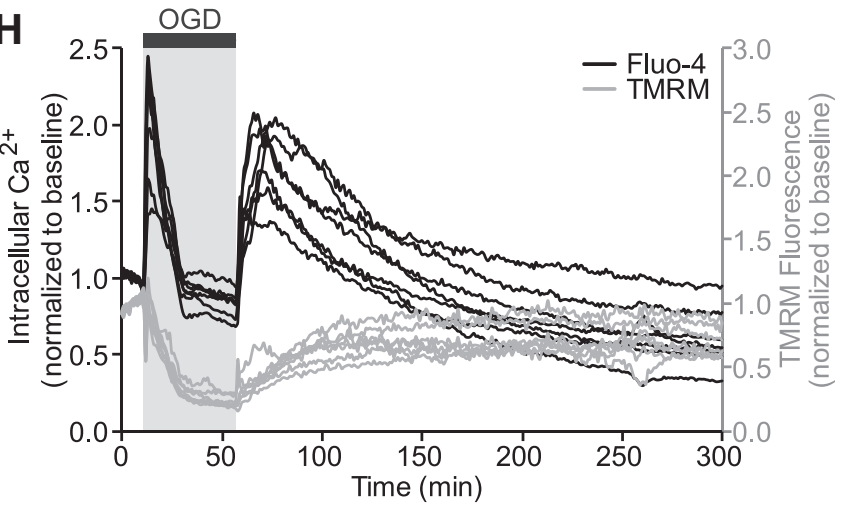

Figure 1. Single-neuron time-lapse imaging experimental models of transient NMDA receptor-mediated excitotoxicity and OGD. $A$, Transient glutamate excitotoxicity experimental treatment paradigm. After 7-9 DIV, CGNs were mounted on a confocal microscope and exposed to glutamate (100 $\mu \mathrm{M})$ and its coagonist glycine (10 $\mu \mathrm{m})$ for $10 \mathrm{~min}$. Neurons were imaged for up to $16 \mathrm{~h}$ after treatment. $\boldsymbol{B}$, Cell death after $24 \mathrm{~h}$ (as quantified by pyknotic nuclei counts) was significantly higher in neurons transiently exposed to glutamate (Glut) compared with sham conditions ( $p=4 \times$ $10^{-4}$ ). C, Single-cell fluorescence intensity traces of CGNs stained with Fluo-4 (black lines, a reporter for intracellular calcium) and TMRM (gray lines, a reporter of mitochondrial membrane potential). Illustrative traces of seven cells are representative of at least three independent experiments. Glutamate rapidly and transiently increased intracellular calcium. Mitochondrial membrane potential decreased throughout glutamate exposure, and more slowly returned to baseline. The dotted trace shows a CGN undergoing delayed calcium deregulation and mitochondrial membrane depolarization (rapid loss of TMRM signal). Two cells were removed from the right hand trace for clarity. The thick gray bar indicates time of glutamate exposure. D, Transient NMDA-mediated excitotoxicity experimental treatment paradigm. In a model similar to that of CGNs exposed to glutamate, $7-9$ DIV Corticals were exposed to NMDA (100 $\mu \mathrm{M})$ and glycine (10 $\mu \mathrm{M})$ for 5 min. $\boldsymbol{E}$, Levels of cell death were significantly higher in Corticals exposed to NMDA compared with those exposed to sham conditions $\left(p=8 \times 10^{-14}\right)$. Single-cell Fluo-4 and TMRM traces of Corticals exposed to NMDA have been described previously (D'Orsi et al., 2012).F, OGD experimental treatment paradigm. The 7-9 DIV Corticals were mounted on a confocal microscope, and exposed to hypoxic and hypoglycaemic conditions for $45 \mathrm{~min}$, as described in the Materials and Methods section. $G$, Levels of cell death were significantly higher in neurons exposed to $0 G D$ compared with those exposed to sham conditions $\left(p=4 \times 10^{-12}\right)$. $\boldsymbol{H}$, Single-cell fluorescence intensity traces of Corticals stained with Fluo-4 (black lines) and TMRM (gray lines). The onset of OGD induced a transient increase in intracellular calcium levels and mitochondrial membrane depolarization. Restoration of normoxic/normoglycemic conditions induced a second transient increase in intracellular calcium, and a recovery of mitochondrial membrane potential. Illustrative traces of seven cells are representative of three independent experiments. The thick gray bar indicates the time of OGD.

We next used the ATeam reporter to monitor cytosolic ATP levels in single neurons undergoing transient excitotoxicity. Strikingly, we detected a rapid ATP depletion in CGNs during glutamate stimulation followed by a similarly rapid recovery to baseline (to $\pm 10 \%$ of baseline fluorescence). ATP levels subsequently remained constant until depolarization, when a rapid drop in ATP levels indicated extensive failure of ATP production and neuronal death (Fig. 2C,D). ATP levels in Corticals behaved similarly during and after transient NMDA stimulation (Fig. 2E), and were also depleted during OGD (Fig. 2F). Recovery after stimulus termination, however, was incomplete in the OGD treatment paradigm. While ATP levels completely recovered within 10 min of stimulus termination in both CGNs exposed to glutamate (Fig. $2 \mathrm{G}$ ) and Corticals exposed to NMDA (Fig. 2H), ATP remained significantly lower than baseline in Corticals even up to 60 min after the termination of OGD (Fig. 2I).

\section{ATP recovery after transient glutamate exposure involves active mitochondria}

Mitochondria are the major source of neuronal ATP production, and their function can be severely compromised by excessive calcium buffering, such as during excitotoxicity (Nicholls and Budd, 2000). Interestingly, however, our data demonstrated that cytosolic ATP levels recovered significantly earlier than the TMRM signal (data not shown). Utilizing TMRM as a reporter of mitochondrial function, this suggested that, despite some form of remnant mitochondrial dysfunction, CGNs were nevertheless able to completely restore cytosolic ATP. To investigate whether mitochondria were capable of participating in this rapid ATP recovery subsequent to glutamate exposure, we preincubated CGNs in glucose-free buffer supplemented with Na pyruvate (2 $\mathrm{mm}$ ), bypassing glycolysis and providing substrate directly to the mitochondria. Cytosolic ATP levels in these cells were depleted but again recovered rapidly after glutamate exposure (Fig. 3A). Similar to CGNs in buffer containing glucose only $(15 \mathrm{~mm})$, ATP recovery in glucose-free buffer was complete within $10 \mathrm{~min}$ after termination of glutamate stimulation (Fig. 3B, left). Competitive inhibition of glycolytic ATP production via 2-DG pretreatment attenuated this rapid recovery, although ATP levels still recovered to $\sim 80 \%$ of preglutamate levels within $10 \mathrm{~min}$ (Fig. $3 B$, right). These data suggested that ATP production through glycolysis was not required for the recovery of cytosolic ATP levels. 
A

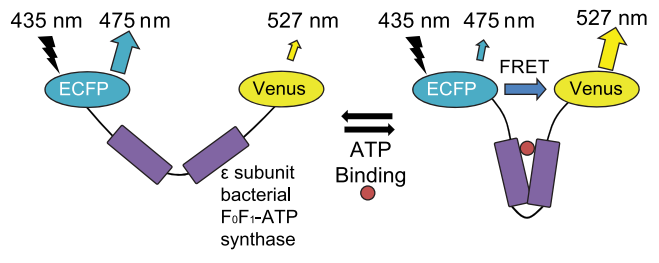

C

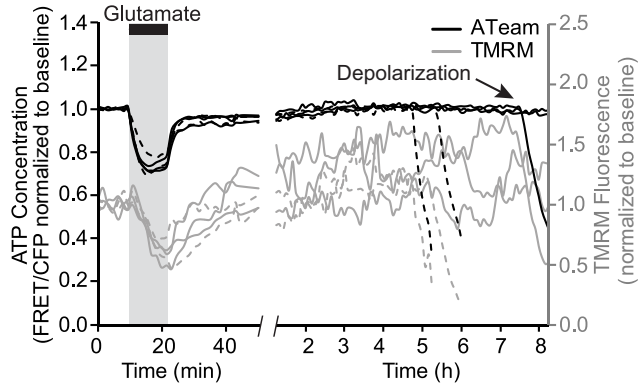

E
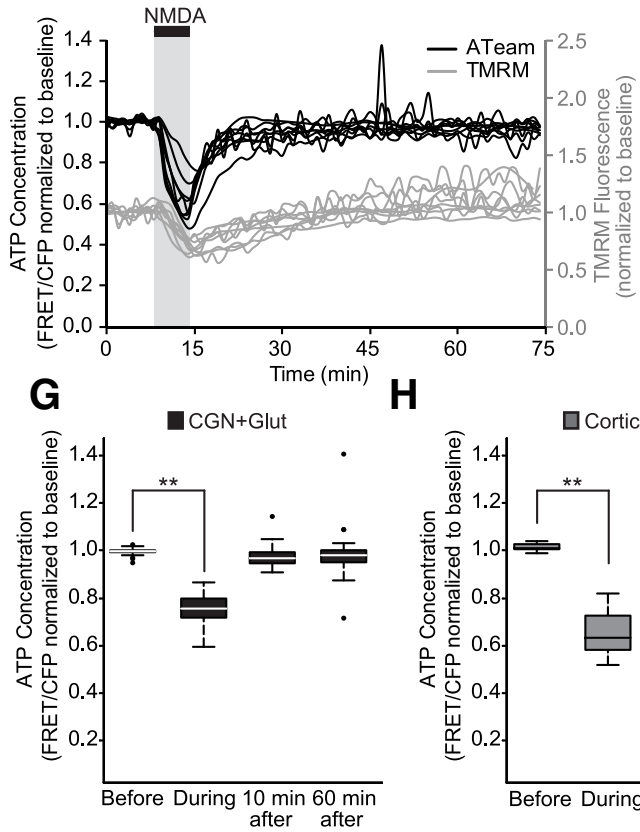

B

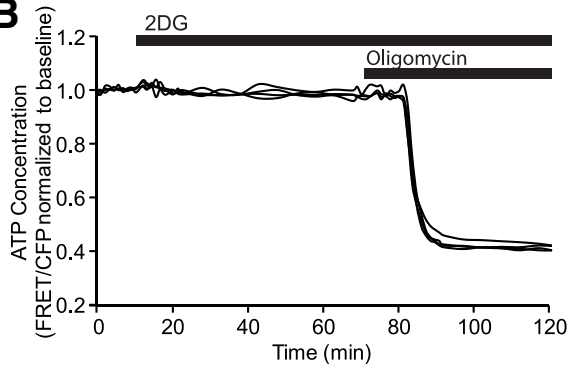

D

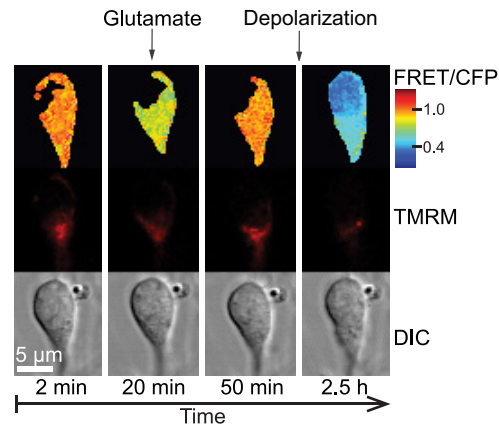

F

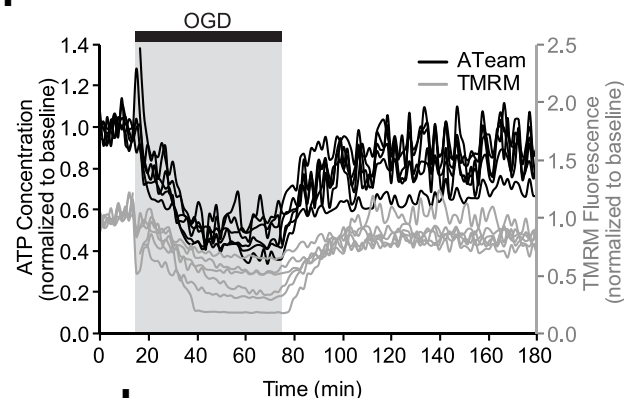

H

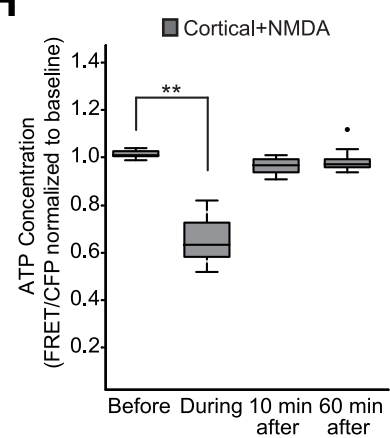

I

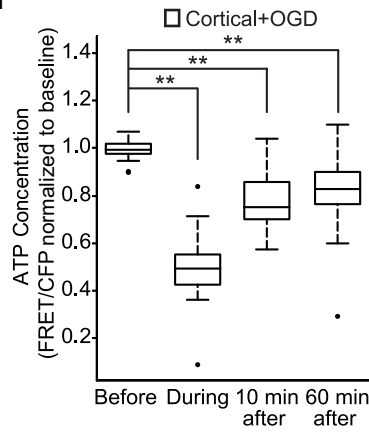

Figure 2. ATP is depleted but rapidly recovers in two experimental models of transient excitotoxicity and this recovery is incomplete after OGD. $A, B$, The ATeam FRET-based reporter used in single-cell experiments is a reporter of intracellular ATP concentration. $\boldsymbol{A}$, Binding of ATP to the $\varepsilon$-subunit of the bacterial $F_{0} / F_{1}$-ATP synthase linker protein increases the fluorescence ratio of FRET/CFP [illustration adapted from Imamura et al., 2009 (permission to publish received from authors)]. $\boldsymbol{B}$, Control experiments demonstrated the complete collapse of ATeam ratiometric fluorescence, to $\sim 40 \%$ of baseline, only after the inhibition of both glycolytic (2-DG) and mitochondrial ATP production (oligomycin), confirming the suitability of the ATeam probe for tracking intracellular ATP levels in our system. C, Single-cell fluorescence ratio and intensity traces of (GNs transfected with the ATeam FRET-based reporter of ATP concentration (black lines), stained with TMRM (gray lines), and exposed to glutamate for 10 min (thick gray bar). Illustrative traces of five cells are representative of five independent experiments. Depolarization was closely accompanied by a rapid decrease of ATP concentration. Tracked neurons were classified as nonsurvivors (dotted traces) if they depolarized within $6 \mathrm{~h}$, and survivors if they remained viable beyond this time (Fig. 6). D, Fluorescence and differential interference contrast (DIC) images of a representative (GN, transiently transfected with the ATeam FRET-based reporter, before, during, and after glutamate exposure, and after mitochondrial membrane depolarization. Color scale represents ratiometric fluorescence values normalized to baseline. $\boldsymbol{E}$, Single-cell fluorescence ratio and intensity traces of Cortical neurons transfected with the ATeam FRET-based reporter of ATP concentration (black lines), stained with TMRM (gray lines) and exposed to NMDA for 5 min (thick gray bar). Illustrative traces of nine cells are representative of three independent experiments. $\boldsymbol{F}$, Single-cell fluorescence ratio and intensity traces of Corticals transfected with the ATeam FRET-based reporter of ATP concentration (black lines), stained with TMRM (gray lines) and exposed to $0 G D$ for $45 \mathrm{~min}$ (thick gray bar). Illustrative traces of six cells are representative of four independent experiments. G-I, Intracellular ATP concentration before, during, and 10/60 min after exposure to glutamate ( 29 cells from five experiments; $\boldsymbol{G}$ ), NMDA ( 13 cells from three experiments; $\boldsymbol{H}$ ), and 0 GD ( 20 cells from four experiments; $\boldsymbol{I}$ ). $\boldsymbol{G}, \boldsymbol{H}$, ATP was significantly depleted during glutamate and NMDA exposure $\left(p=4 \times 10^{-14}\right.$ and $p=1 \times 10^{-4}$, respectively) and recovered within 10 min. $I$, ATP was significantly depleted during $0 \mathrm{GD}\left(p=2 \times 10^{-7}\right)$ but did not completely recover within 60 min after treatment ( $p=1 \times 10^{-5}$ and $p=6 \times 10^{-5}$ for 10 and 60 min time points, respectively). 
A

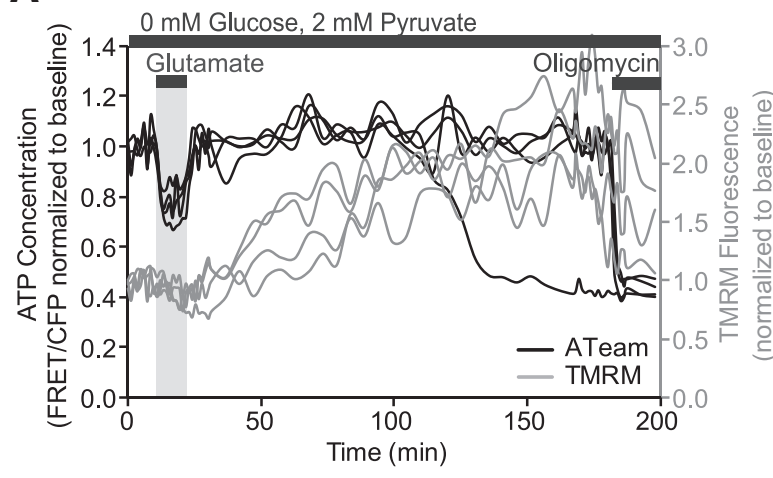

C

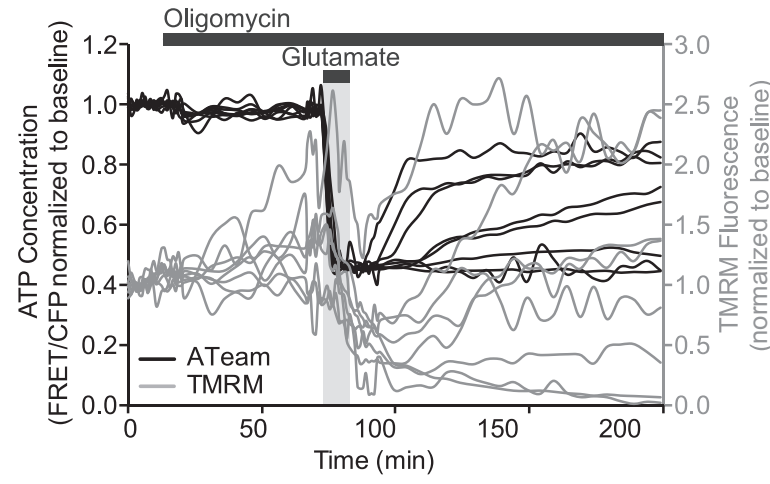

E

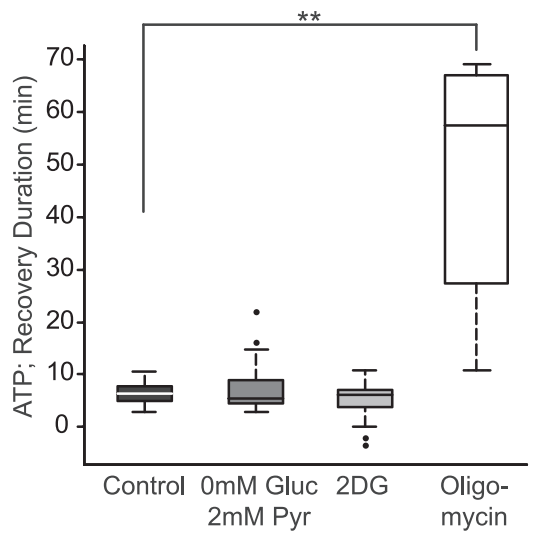

B

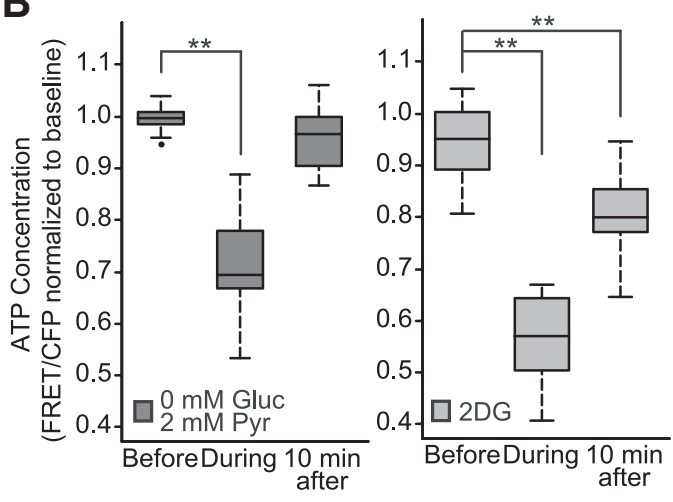

D

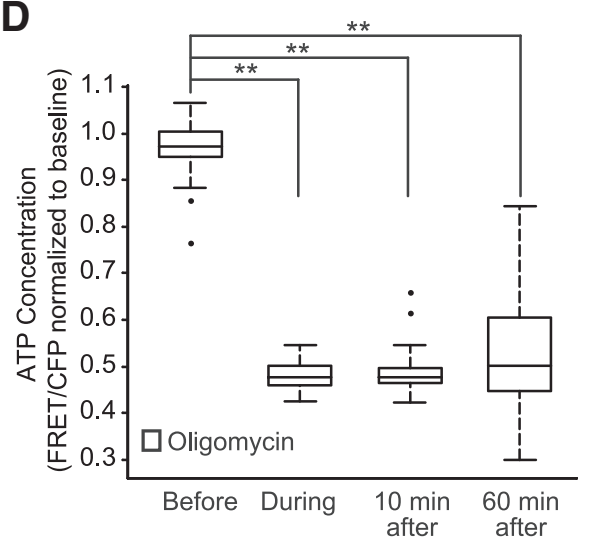

$\mathbf{F}$

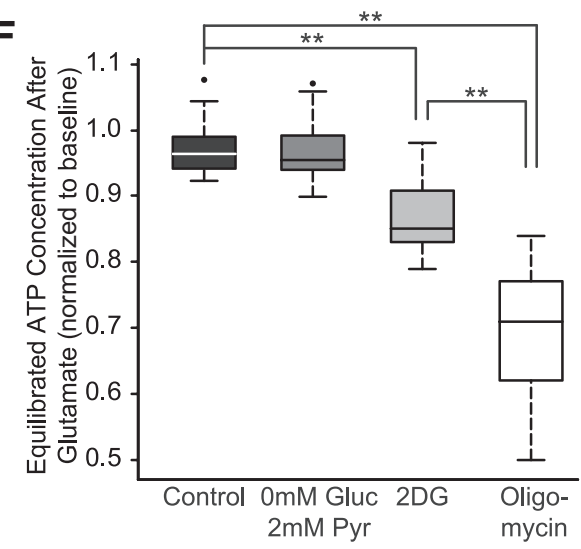

Figure 3. Mitochondrial ATP production is required to rapidly restore ATP to baseline levels after excitotoxic stress. $A, B$, Removal of glucose from the buffer [with supplementation of 2 mm pyruvate (Pyr)] did not prevent the rapid postglutamate recovery of cytosolic ATP. A, ATeam fluorescence ratio and TMRM average fluorescence intensity traces of CGNs transfected with the ATeam probe, incubated in glucose-free buffer supplemented with pyruvate and exposed to glutamate for $10 \mathrm{~min}$. Illustrative traces of four cells representative of three independent experiments. Subsequent addition of oligomycin completely depleted ATP levels. B, ATP levels of CGNs in glucose-free media supplemented with 2 mm pyruvate were significantly depleted during glutamate exposure (17 cells from three experiments; $p=2 \times 10^{-6}$ ) but recovered within $10 \mathrm{~min}$, similar to CGNs in $15 \mathrm{~mm}$ glucose. CGNs were also treated with 2-DG to competitively inhibit glycolytic ATP production. ATP levels in these CGNs recovered but remained significantly depleted $10 \mathrm{~min}$ after glutamate exposure ( 23 cells from three experiments; $p=2 \times 10^{-8}$ and $p=4 \times 10^{-6}$ before compared with subsequent time points). Time points on the $x$-axes refer to glutamate treatment. $\mathbf{C}-\boldsymbol{F}, 0$ ligomycin, an inhibitor of ATP synthase, did not induce observable changes in resting ATP concentration, but significantly exacerbated subsequent glutamate-induced ATP depletion, and inhibited the duration and extent of post-glutamate recovery. $C$, ATeam fluorescence signal and TMRM average fluorescence intensity traces of CGNs treated with oligomycin ( $10 \mu \mathrm{m}$, solid black bar) and subsequently exposed to glutamate for 10 min (thick gray bar). Illustrative traces of eight cells are representative of three independent experiments. D, ATP levels in oligomycin-treated CGNs before, during, and 10 and 60 min after glutamate exposure. Oligomycin significantly exacerbated ATP depletion during glutamate exposure and inhibited the rapid recovery (31 cells from three experiments; $p=9 \times 10^{-11}, 8 \times 10^{-11}, 5 \times 10^{-11}$ comparing before with subsequent time points). $\boldsymbol{E}, \boldsymbol{F}$, Some oligomycin-treated (GNs were capable of partially restoring ATP levels after glutamate exposure. In these cells, however, ATP recovered significantly slower $\left(p=2 \times 10^{-7} ; \boldsymbol{F}\right)$ and to a lesser extent $\left(p=2 \times 10^{-7} ; \boldsymbol{F}\right)$ than cells exposed to glutamate alone (Control). The extent of ATP recovery was also affected by $2-\mathrm{DG}\left(p=4 \times 10^{-8}\right)$, although not to the same extent as with oligomycin ( $p=3 \times 10^{-5}$ comparing 2-DG with oligomycin).

To further investigate the role of mitochondrial ATP production in this process, we treated CGNs in $15 \mathrm{~mm}$ glucose with oligomycin $(2 \mu \mathrm{g} / \mathrm{ml})$, an inhibitor of ATP synthase and therefore of mitochondrial ATP production, 60 min before glutamate exposure. Oligomycin had no measureable effect on ATP concentration in neurons at rest (Fig. $3 C$ ), confirming that glycolysis is sufficient to maintain ATP levels in these circumstances (Budd and Nicholls, 1996; Vergun et al., 2003; Jekabsons and Nicholls, 
2004; Khodorov et al., 2012). In contrast, the glutamate-induced ATP depletion was markedly exacerbated in oligomycin-treated neurons (Fig. 3C), suggesting that mitochondrial ATP production minimizes the glutamate-induced energetic crisis. Strikingly, the rapid ATP recovery seen in cells exposed only to glutamate was not observed in oligomycin-treated cells (Fig. 3C,D). Nevertheless, as seen in Figure 3C, some oligomycin-treated CGNs (14 of 34 cells from three independent experiments), partially recovered ATP levels following glutamate exposure. This recovery was significantly slower (Fig. 3E), and final ATP levels were significantly lower (Fig. $3 F$ ), than in CGNs treated with glutamate alone (Control), in those where glucose was substituted with pyruvate, or in those where 2-DG pretreatment inhibited glycolysis. A less prominent reduction in ATP recovery was also observed in CGNs pretreated with 2-DG, which may be in part due to the downstream effect of glycolysis inhibition on mitochondrial ATP production, as these cells had not been supplemented with pyruvate. Finally, oligomycin addition after glutamate exposure did not affect ATP levels in CGNs in $15 \mathrm{~mm}$ glucose (data not shown), suggesting that glycolysis was sufficient to maintain baseline ATP levels once recovery had been achieved. Together, these data suggested that mitochondrial ATP production played a key role in rapidly restoring ATP levels to baseline.

\section{AMPK activity in single neurons is rapidly and transiently} increased during excitotoxicity, and is decreased during OGD AMPK is involved in the maintenance of bioenergetic homeostasis (Hardie et al., 2012) and, hence, may regulate energetic recovery after an excitotoxic or OGD-induced energetic crisis. We and others have previously demonstrated that ATP depletion during excitotoxic injury increases protein levels of active, threonine172-phosphorylated AMPK during glutamate excitotoxicity, indicating higher AMPK activity (McCullough et al., 2005; Weisová et al., 2009; Concannon et al., 2010; Davila et al., 2012). To directly monitor AMPK activity in our single-cell system, we used a FRET-based reporter of AMPK activity. The probe incorporates a synthetic peptide that can be phosphorylated by AMPK, altering the probe conformation and increasing FRET, and therefore the ratiometric fluorescent signal (Tsou et al., 2011; Fig. 4A). As a positive control for this probe, we confirmed that the administration of the AMP "mimetic" AICAR or exposure of 2-DG to CGNs and Corticals, respectively, increased the AMPKAR ratiometric fluorescence signal, consistent with the AMPK elevation after these drug exposures observed by other measurements (Fig. $4 B)$. Using this probe, we then found that AMPK activity rapidly and transiently increased during glutamate treatment in CGNs (Fig. 4C,D) and during NMDA treatment in Corticals (Fig. 4E). Notably, in some neurons, AMPK activity began to decrease before termination of the excitotoxic stimulus, suggesting that AMPK activity was no longer required in these cells. In contrast, AMPK activity levels were slightly decreased during OGD treatment in Corticals (Fig. 4F). In all treatment paradigms, AMPK activity rapidly and completely returned to baseline within 10 min after stimulus termination (Fig. 4G-I).

\section{Intracellular glucose increases after excitotoxicity}

The rapid recovery of ATP levels indicated that neurons were well capable of satisfying the metabolic requirements for survival in the acute phase after transient excitotoxic insults. However, sources of energy are needed to cope with the excitotoxic injury, and to restore the ionic and metabolic balance. Indeed, previous data from our group demonstrated increased glucose uptake during excitotoxic injury in CGNs in a population-based bio- chemical assay (Ward et al., 2007), and suggested a role for AMPK-induced GLUT-3 surface expression in mediating this effect (Weisová et al., 2009). We therefore wanted to investigate intracellular glucose levels in our single-cell system, and transiently transfected neurons with a FRET-based reporter of intracellular glucose concentration, glucose-FRET (Takanaga et al., 2008; Fig. 5A). To confirm the sensitivity of the reporter to glucose, we increased the extracellular glucose concentration and detected a corresponding stepwise increase in the emitted glucose-FRET ratiometric fluorescence signal (Fig. 5B).

We initially anticipated that glucose consumption via glycolysis would be increased during excitotoxicity to address the ATP depletion, and that this demand would outweigh any potential elevation in glucose uptake. However, in contrast to this expected decrease in intracellular glucose, we consistently observed a glutamate-mediated increase in CGNs (Fig. 5C-E). As an additional control experiment, we investigated responses of neurons transfected with a mutated version of the glucose-FRET reporter, where one residue of the glucose-galactose binding protein had been mutated to reduce the sensitivity of the reporter to glucose (termed control-FRET herein; Takanaga et al., 2008). We confirmed that the control-FRET reporter was insensitive to changes in extracellular glucose at the levels used in our system (15 mM; data not shown). Control-FRET-transfected CGNs exposed to glutamate for $10 \mathrm{~min}$ exhibited a slight increase in the emitted ratiometric fluorescence during the glutamate exposure period only, which may be due to glutamate-induced cellular alterations other than glucose, but otherwise showed no response (Fig. $5 F$ ). To further corroborate the finding of a glutamate-induced increase in intracellular glucose during and after glutamate exposure, we measured intracellular glucose in neuronal populations using an assay based on the conversion of glucose to a fluorometric product via the glucose oxidase enzyme. This assay confirmed that glucose was indeed increased during and shortly after glutamate stimulation in CGNs incubated in 15 mM glucose (Fig. 5G).

We also detected an elevation of intracellular glucose in single Corticals after exposure to NMDA, with kinetics similar to CGNs exposed to glutamate (Fig. $5 H, I$ ). Interestingly, OGD did not significantly affect the glucose-FRET fluorescence signal (Fig. $5 J, K)$. Together, these results indicated that abundant intracellular glucose was available to restore ATP after excitotoxic insults, and that substrate depletion was not rate limiting for ATP production in our system.

\section{The excitotoxicity-induced increase in intracellular glucose originates from extracellular, AMPK-dependent glucose uptake and intracellular glucose mobilization}

We previously linked glutamate-mediated glucose uptake to elevated AMPK activity (Weisová et al., 2009). Our single-cell data here indeed confirmed a concomitance of transient intracellular glucose, elevated AMPK activity in both excitotoxicity models, and, in turn, demonstrated that no significant glucose elevation was observed during OGD, where AMPK activity was decreased. Hence, to further investigate the role of AMPK activity in glucose levels during excitotoxicity, we treated CGNs with CompC (10 $\mu \mathrm{M})$, an ATP-competitive inhibitor of AMPK. The initial administration of CompC decreased the glucose-FRET signal, confirming that AMPK regulated intracellular glucose concentration in neurons at rest (Fig. 6A). Of note, the glutamate-induced increase in the glucose-FRET signal was reduced in the presence of CompC (compare Figs. 6B, 5E), yet the signal nevertheless remained elevated beyond stimulus termination (Fig. 6B). This 
A

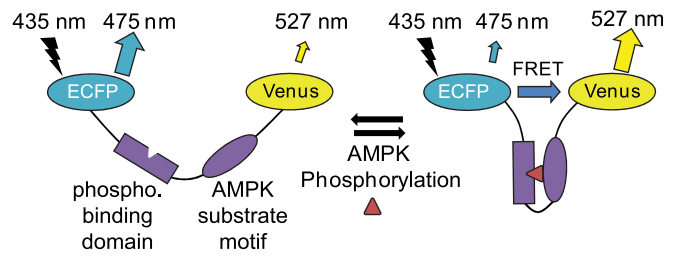

C

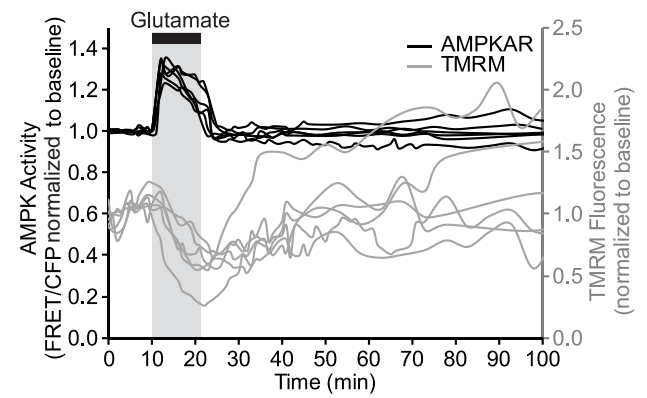

E

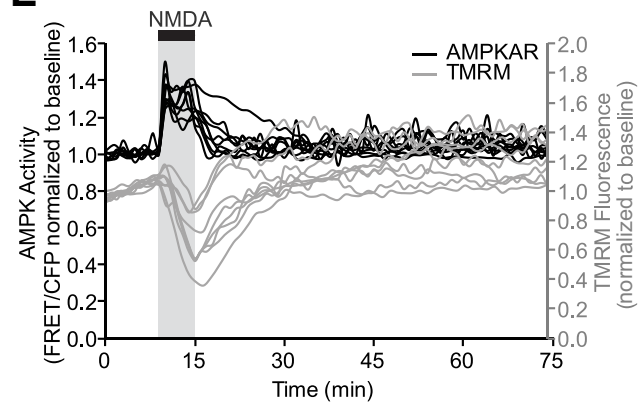

B

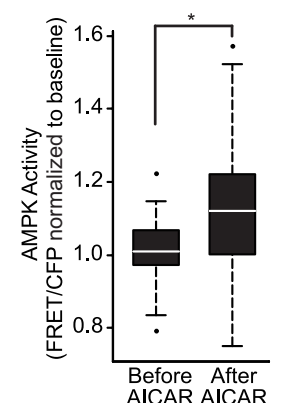

D

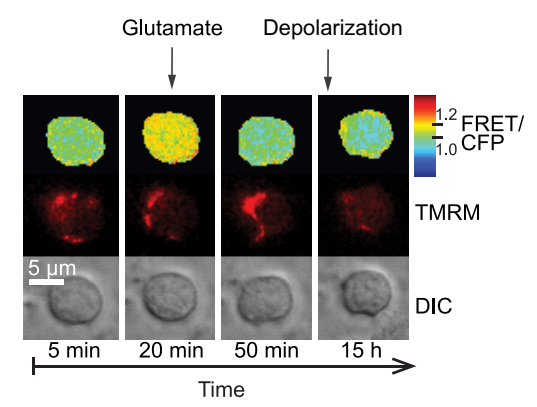

F

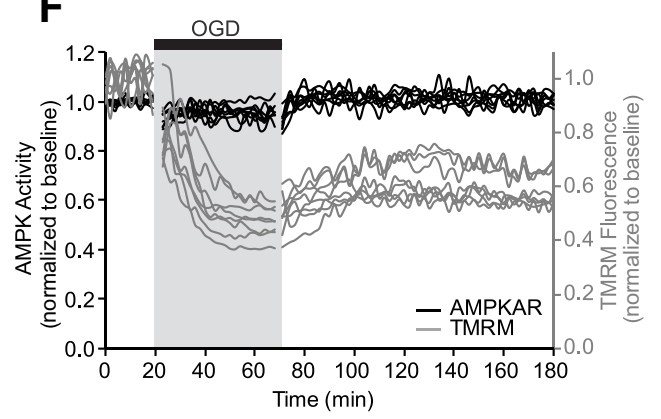

G

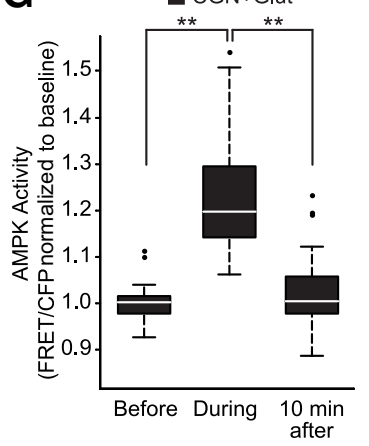

H

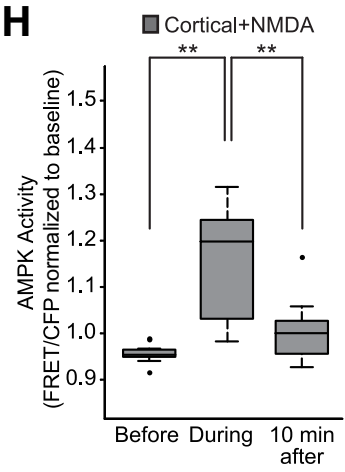

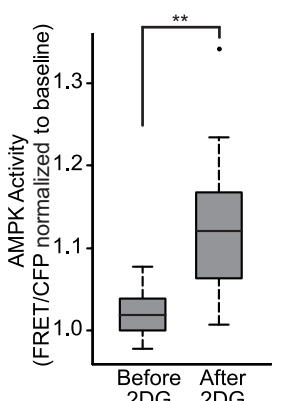

After
2DG

$(15 \mathrm{~min})$

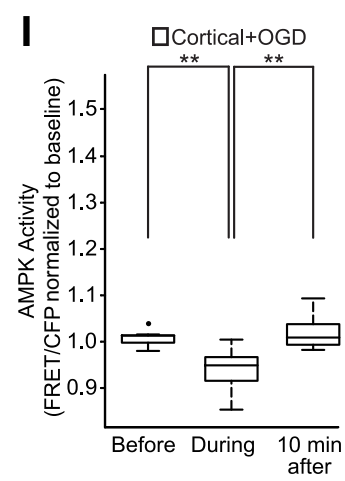

Figure 4. AMPK activity increases in single neurons during transient glutamate- and NMDA-mediated excitotoxicity, and decreases during OGD. $A, B$, The AMPKAR FRET-based reporter used in single-cell experiments is a reporter of AMPK activity. $A$, Phosphorylation of the synthetic peptide by AMPK increases the fluorescence ratio of FRET/CFP, indicating an increase in AMPK activity (illustration adapted from Tsou et al., 2011 [license to publish from Elsevier (\#3415451067680)]). B, Exposure of (GNs to $2.5 \mathrm{~mm} \mathrm{AICAR} \mathrm{(31} \mathrm{cells} \mathrm{from} \mathrm{three} \mathrm{experiments)} \mathrm{or} \mathrm{cortical} \mathrm{neurons} \mathrm{to} 12 \mathrm{~mm}$ 2-DG (17 cells from three experiments) increased the fluorescence ratio of the AMPKAR reporter, indicating its suitability for the monitoring of AMPK activity levels in CGNs and Corticals ( $p=2 \times$ $10^{-3}$ and $p=9 \times 10^{-5}$, respectively). $\boldsymbol{C}, \boldsymbol{D}$, Glutamate induced a transient increase in AMPK activity. C, Single-cell ratiometric fluorescence signal and intensity traces of (GNs transfected with AMPKAR (black lines), stained with TMRM (gray lines) and exposed to glutamate for 10 min (thick gray bar). Illustrative traces of six cells are representative of four independent experiments. $\boldsymbol{D}$, Fluorescence and differential interference contrast (DIC) images of a representative CGN, transiently transfected with AMPKAR, before, during, and after glutamate exposure, and after mitochondrial membrane depolarization. Color scale represents ratiometric fluorescence values normalized to baseline. $\boldsymbol{E}$, NMDA also induced a transient increase in AMPK activity. Single-cell fluorescence ratio and intensity traces of cortical neurons transfected with AMPKAR (black lines), stained with TMRM (gray lines) and exposed to NMDA for 5 min (thick gray bar). Illustrative traces of nine cells are representative of four independent experiments. $F$, AMPK activity decreased during OGD. Single-cell fluorescence intensity traces of GGNs transfected with AMPKAR (black lines), stained with TMRM (gray lines), and exposed to OGD for 45 min (thick gray bar). Illustrative traces of eight cells representative of two independent experiments. G-I, Box-plots of AMPK activity before, during and 10 min after exposure to glutamate ( 31 cells from four experiments; $\boldsymbol{G}$ ), NMDA (20 cells from four experiments; $\boldsymbol{H}$ ), and OGD (11 cells from two experiments; $\boldsymbol{I})$. $\boldsymbol{G}, \boldsymbol{H}$, AMPK activity was significantly elevated during glutamate and NMDA exposure $\left(p=6 \times 10^{-11}\right.$ and $p=1 \times 10^{-7}$, respectively) and recovered within $10 \mathrm{~min}\left(p=6 \times 10^{-9}\right.$ and $p=2 \times 10^{-4}$, respectively). $I$, In contrast, AMPK activity was transiently decreased during $\operatorname{OGD}\left(p=1 \times 10^{-3}\right)$ but again returned to baseline within $10 \mathrm{~min}\left(p=2 \times 10^{-3}\right)$. 
A
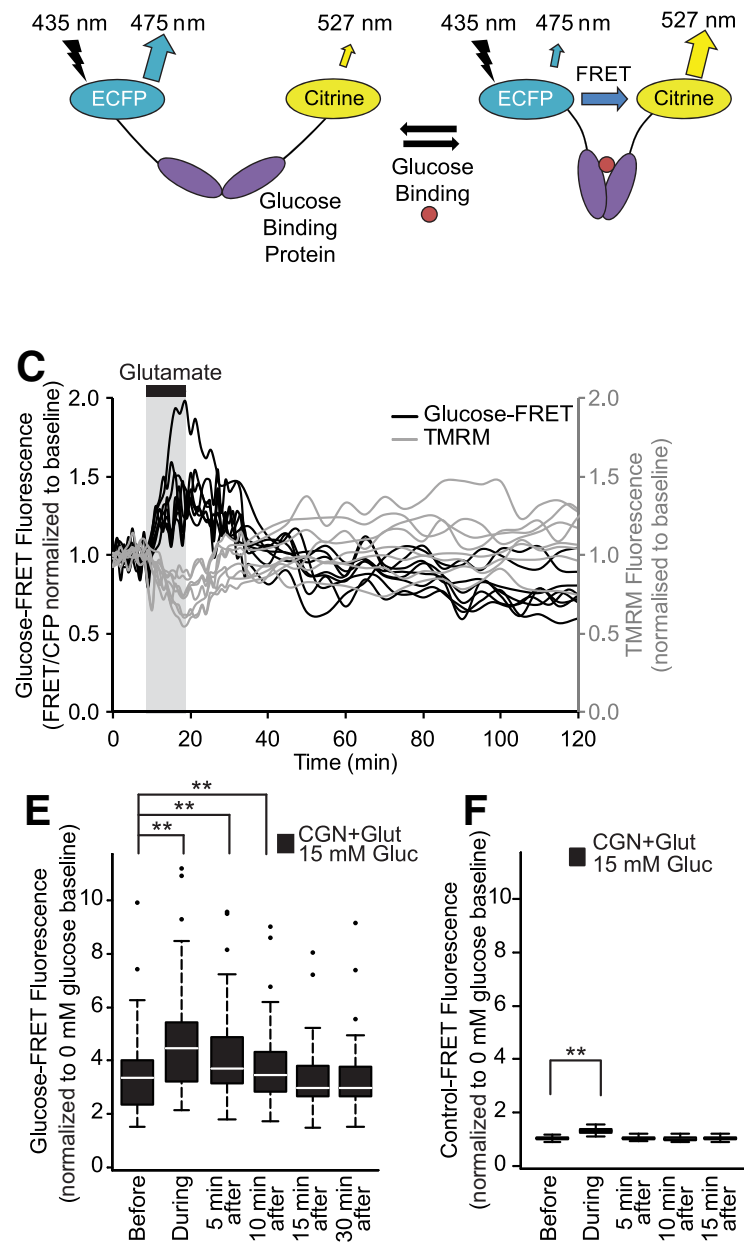

H
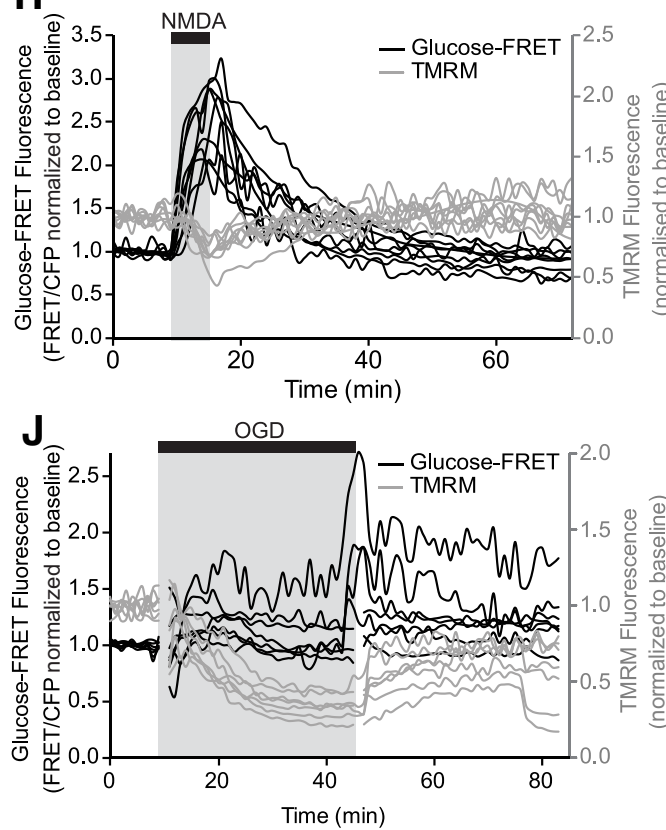

B

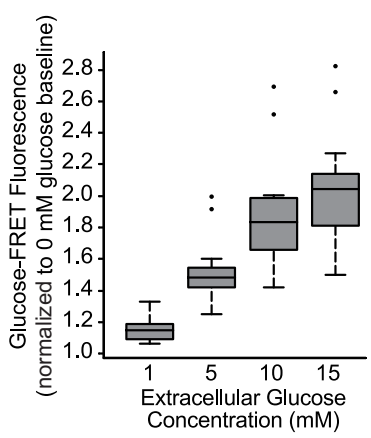

D
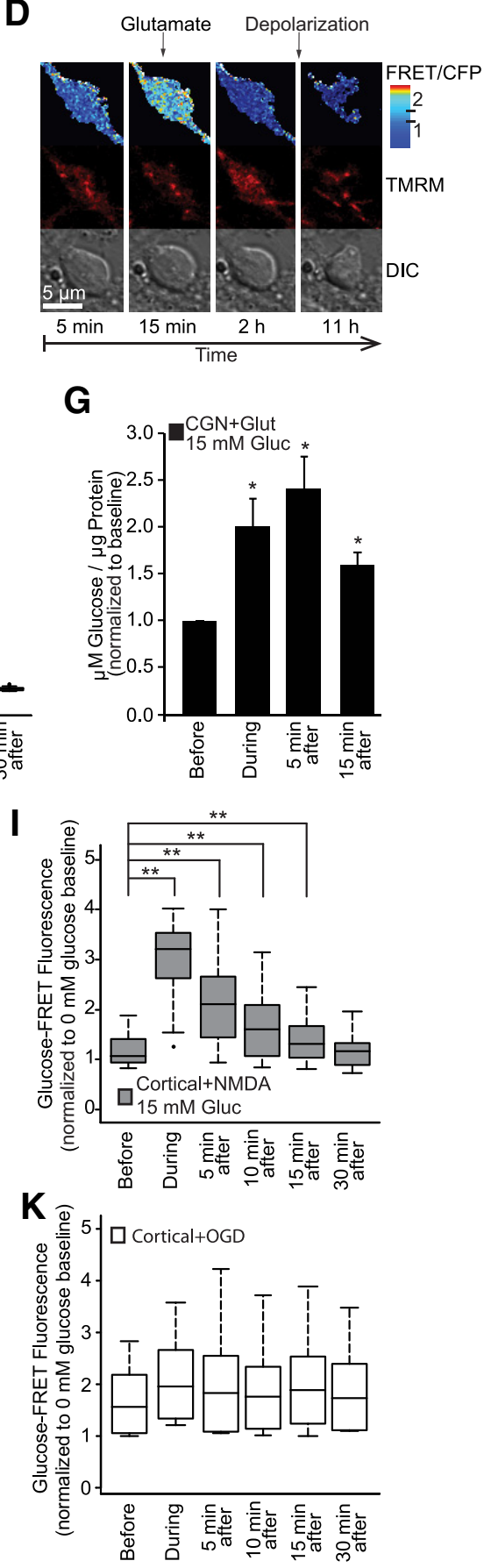

Figure 5. Intracellular glucose is increased in single neurons after transient NMDA receptor-mediated excitotoxicity. $\boldsymbol{A}, \boldsymbol{B}$, The glucose-FRET-based reporter used in single-cell experiments is a reporter of intracellular glucose concentration. $\boldsymbol{A}$, Binding of glucose to the glucose- galactose binding protein increases the fluorescence ratio of FRET/CFP (Takanaga et al., 2008). $\boldsymbol{B}$, Increasing the extracellular glucose concentration increased the emitted fluorescence ratio in cortical neurons, indicating the suitability of the glucose-FRET probe to report intracellular glucose levels. $\boldsymbol{C}, \boldsymbol{D}$, Glutamate induced a transient increase in intracellular glucose. C, Fluorescence intensity traces of individual CGNs transfected with the glucose-FRET reporter (Figure legend continues.) 
suggested that, while the glutamate-mediated increase in intracellular glucose was partially AMPK mediated, there nevertheless remained an AMPK-independent fraction.

We next wanted to investigate whether the excitotoxicityinduced increase in intracellular glucose was entirely driven by extracellular sources and therefore incubated CGNs in glucosefree media supplemented with pyruvate. We indeed measured a reduced increase in the glucose-FRET signal, confirming that the observed glucose increase was primarily coming from extracellular sources (compare Figs. 6C, $5 E$ ). Interestingly, however, the glucose-FRET signal still remained elevated, even beyond stimulus termination, in the absence of extracellular glucose, pointing to an additional and intracellular glucose source for disposal during excitotoxic conditions. Notably, as measured by TMRM signal, these cells showed a recovery of the mitochondrial membrane potential to baseline (to an average of $1.2 \pm 0.13$ after 10 $\mathrm{min}$ ), which was comparable to that observed in neurons maintained in $15 \mathrm{~mm}$ glucose $(1.09 \pm 0.05$ after $10 \mathrm{~min})$, and remained viable for the duration of the experiments. Similar glucose and TMRM responses were also recorded after the removal of both glucose and pyruvate from the extracellular media, giving evidence that any such internal glucose source may be sufficient to maintain mitochondrial function and neuronal viability after excitotoxic injury (data not shown).

Interestingly, a recent study (Saez et al., 2014) suggested that neurons are able to mobilize glycogen, and that glycogen metabolism was protective in primary neurons exposed to hypoxia. To test this hypothesis, we inhibited glycogen metabolism with two pharmacological compounds, DAB and NJM (inhibitors of glycogen phosphorylase and $\alpha$-glucosidase, respectively). Indeed, this inhibition of glycogen metabolism reduced the degree and the time for which the glucose-FRET signal remained elevated, comparable to those responses measured with the control-FRET probe (compare Figs. $6 D, 5 F$ ), suggesting that glycogen metabolism may contribute to the intracellular glucose increase during excitotoxicity.

\section{$\leftarrow$}

(Figure legend continued.) (black lines), stained with TMRM (gray lines), and exposed to glutamate for 10 min (thick gray bar). Illustrative traces of eight cells representative of three independent experiments. $\boldsymbol{D}$, Fluorescence and differential interference contrast (DIC) images of a representative CGN transiently transfected with the glucose-FRET reporter before, during, and after glutamate exposure, and after mitochondrial membrane depolarization. Color scale represents ratiometric fluorescence values normalized to baseline. $E$, Glucose-FRET signal in CGNs incubated in $15 \mathrm{~mm}$ extracellular glucose before, during, and after glutamate exposure. The FRET signal was normalized to an average of the baseline ratiometric fluorescence signal measured in neurons incubated in $0 \mathrm{~mm}$ glucose. The glucose-FRET signal was significantly increased during and after glutamate exposure ( 32 cells from three experiments; $p=4 \times 10^{-6}, 2 \times$ $10^{-5}$, and 0.02 comparing before to subsequent time points). $\boldsymbol{F}$, The ratiometric fluorescence signal of the control-FRET reporter, a mutated version of the glucose-FRET reporter with reduced glucose sensitivity, was increased only during glutamate exposure (18 cells from two experiments; $\left.p=1 \times 10^{-3}\right)$. $\mathbf{G}$, Measurement of intracellular glucose levels at the population level with a glucose-oxidase fluorometric assay confirmed an increase in glucose levels during and after glutamate exposure in CGNs (three independent experiments; $p=0.04,0.03,0.02$ comparing before to subsequent time points). $\boldsymbol{H}$, Fluorescence ratio and intensity traces of individual cortical neurons transfected with the FRET-based reporter of intracellular glucose concentration (black lines), stained with TMRM (gray lines) and exposed to NMDA for 5 min (thick gray bar). Illustrative traces of nine cells representative of three independent experiments. $I$, The glucose-FRET ratiometric signal was significantly increased during and up to 15 min after NMDA exposure ( 19 cells from three experiments; $p=1 \times 10^{-3}, 1 \times 10^{-3}, 1 \times$ $10^{-3}$, and 0.03 comparing before to subsequent time points). J, Fluorescence ratio and intensity traces of individual Corticals transfected with the glucose-FRET reporter (black lines), stained with TMRM (gray lines), and exposed to OGD for 45 min (thick gray bar). Illustrative traces of seven cells representative of three independent experiments. $\boldsymbol{K}$, The glucose-FRET signal was not significantly altered during or after OGD (10 cells from three experiments).

\section{Neurons that show a prolonged intracellular glucose elevation die earlier}

We finally were interested whether the acute responses observed during excitotoxicity were related to the duration of neuronal survival after the insult. We therefore distinguished neurons with sustained TMRM signal $>6 \mathrm{~h}$ after glutamate exposure, designated "survivors," from those with a loss of TMRM within this time ("nonsurvivors"; Fig. 2C, dotted line traces). Interestingly, the area under the ATP curve, the minimum ATP levels, or the speed of ATP recovery did not significantly differ between survivors and nonsurvivors (data not shown). This suggested that, once neurons recovered from the acute injury phase, the extent of the glutamate-induced ATP depletion was not a critical determinant or a correlate of the duration of subsequent survival. Similarly, the extent of AMPK activity did not differ between survivors and nonsurvivors (data not shown).

In contrast to ATP concentration and AMPK activity, we surprisingly found that glucose levels in nonsurvivors recovered significantly slower than in those neurons that remained viable after $6 \mathrm{~h}$ (survivors; Fig. 6E). Indeed, the recovery of glucose levels was significantly slower and showed a higher cell-to-cell heterogeneity than the recovery of ATP concentration and AMPK activity (Fig. $6 F$ ). To further investigate this, we calculated the correlation between the duration of glucose recovery, determined as the time from stimulus termination to recovery to baseline; and the survival time, measured from stimulus termination to loss of TMRM signal. We found that a more rapid return of glucose to baseline, but not other parameters such as maximum glucose amount, significantly correlated with prolonged survival (Spearman correlation coefficient $=-0.52 ; p=0.039$ ). Additionally, neurons that recovered quicker survived longer than those in which recovery lasted longer (Fig. 6G). From these data, we concluded that the plasticity of neurons to rapidly restore glucose homeostasis was a predictor of neuronal survival.

\section{Discussion}

The present study is the first to investigate experimentally, from a single-cell perspective, the behavior of ATP and glucose levels and AMPK activity in single primary neurons undergoing transient NMDA receptor-mediated excitotoxicity or OGD. Our results provide the following findings: using fluorescent protein fusion plasmids based on FRET, we demonstrated in two excitotoxicity models that ATP depletion and recovery to energetic homeostasis, along with AMPK activation, were surprisingly rapid responses. We observed rapid recovery of neuronal ATP even in the absence of extracellular glucose or with inhibition of glycolytic ATP production, demonstrating the remarkable plasticity of neurons in excitotoxic conditions. Of note, we also demonstrated that unimpaired mitochondria were critical for this fast and complete energetic recovery. Using an injury model of OGD, we identified a similarly rapid bioenergetics response, yet with incomplete ATP recovery and decreased AMPK activity. Surprisingly, we also measured a glutamate and NMDA-mediated increase in intracellular neuronal glucose, providing a potential source of energy after glutamate exposure. Further single-cell experiments revealed that this glucose increase originated from extracellular, AMPK-dependent glucose uptake and from intracellular glucose sources, again demonstrating the significant plasticity of primary neurons under excitotoxic conditions. Furthermore, we demonstrated that the ability to more quickly restore glucose levels to baseline was indicative of neuronal survival.

Previous population studies demonstrated that neurons exposed to transient excitoxicity are able to recover ATP levels, 
A
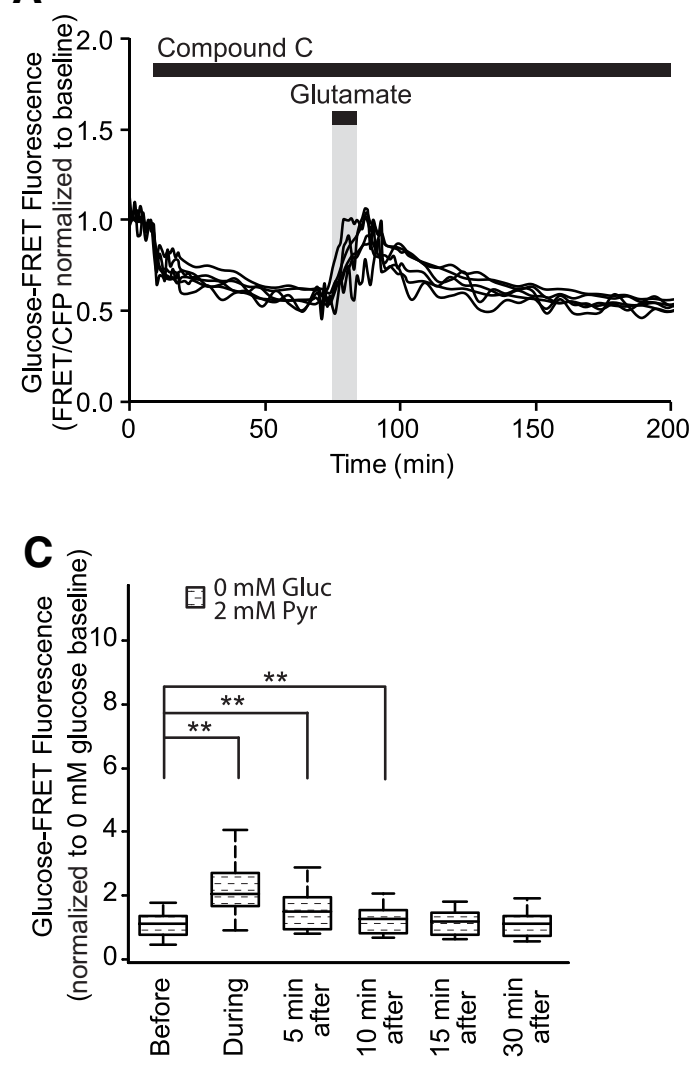

$\mathbf{E}$

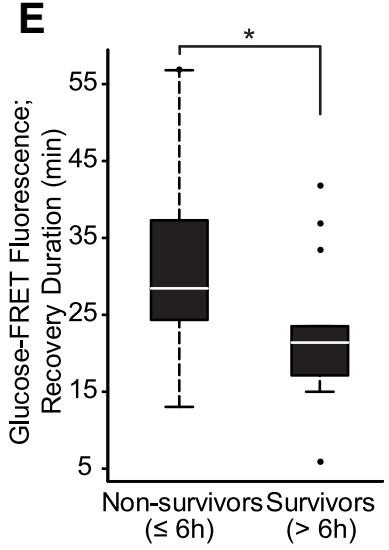

B

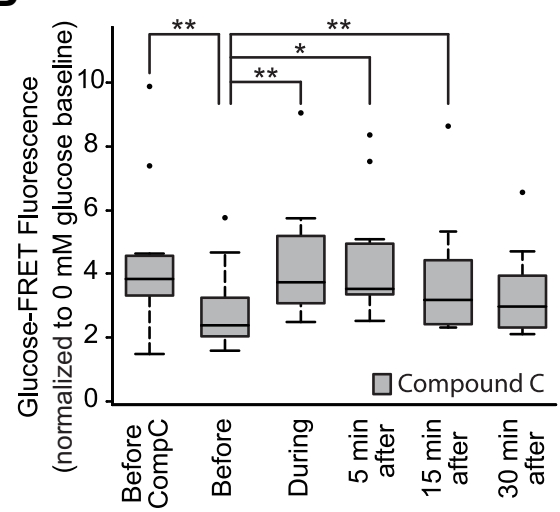

D

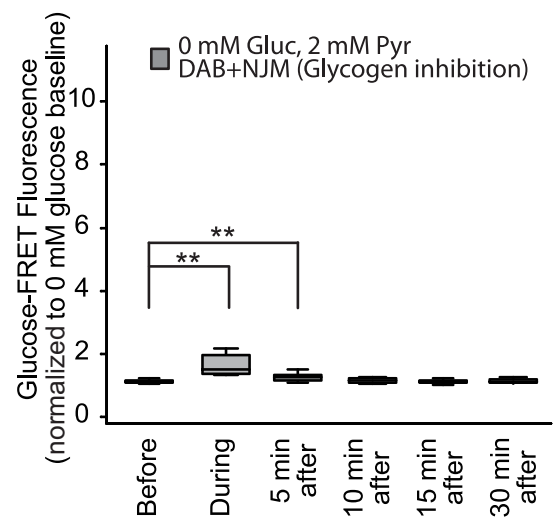

G

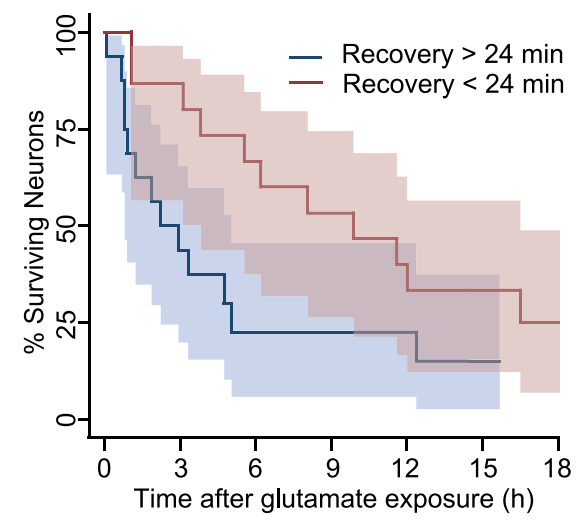

Figure 6. Glutamate-induced glucose elevation originates from extracellular AMPK-dependent glucose uptake and from intracellular glucose mobilization, and glucose-FRET recovery is delayed in CGNs that die earlier. $\boldsymbol{A}$, Single-cell traces of the ratiometric glucose-FRET fluorescence signal in CGNs treated with compound C (10 $\mu \mathrm{M}) 60$ min before glutamate treatment. Illustrative traces of six cells representative of three independent experiments. $\boldsymbol{B}$, Compound (depleted intracellular glucose levels in CGNs at rest $(p=0.004)$, and reduced but did not completely inhibit the subsequent glutamate-induced glucose-FRET ratio increase (11 cells from three experiments, $p=5 \times 10^{-3}, 1 \times 10^{-2}, 5 \times 10^{-3}$ comparing before to subsequent time points). C, CGNs incubated in 0 mM glucose also increased their glucose-FRET signal during and after glutamate exposure (20 cells from four experiments, $p=5 \times 10^{-4}, 4 \times 10^{-4}, 5 \times 10^{-4}$ comparing before to subsequent time points), although this increase was substantially reduced compared with CGNs incubated in $15 \mathrm{~mm}$ glucose (compare with Fig. 5E). D, Inhibition of glycogen metabolism (with DAB plus NJM, inhibitors of glycogen phosphorylase and $\alpha$-glucosidase, respectively) markedly reduced the period for which glucose remained elevated after glutamate stimulation (13 cells from two experiments, $p=1 \times 10^{-3}$ and $5 \times 10^{-3}$, comparing before to subsequent time points). $\boldsymbol{E}$, The glucose-FRET signal recovered significantly slower in cells that survived $>6 \mathrm{~h}$ (survivors; $p=0.027$ ). $\boldsymbol{F}$, The recovery of the glucose-FRET signal took significantly longer than that of ATP $\left(p=6 \times 10^{-12}\right)$ and AMPK activity $\left(p=9 \times 10^{-12}\right)$, as measured by the ATeam and AMPKAR reporters, respectively. G, Kaplan-Meier curve demonstrating that CGNs that recovered their glucose-FRET signal to baseline in $<24$ min survived longer than those whose recovery lasted $>24$ min. The $95 \%$ confidence interval bands are shown.

confirming our single-cell data (Ankarcrona et al., 1995; Ward et al., 2007; Weisová et al., 2009). Population measurements, however, can be skewed by an unsynchronized response, and by contributions from glial cells and dying neurons, resulting in apparently slower kinetics (Loewer and Lahav, 2011). Our experiments showed that after recovery, ATP was maintained at a con- stant level until cells eventually underwent mitochondrial membrane depolarization. Strikingly, ATP levels were recovered, and were subsequently maintained, both in the absence of extracellular glucose and under conditions of glycolysis inhibition, highlighting the ability of neurons to adapt to varying bioenergetic stressors. Indeed, it has been shown that the ATP produc- 
tion machinery can respond quickly to changing conditions (Klingenberg, 2008). Rodriguez-Rodriguez et al. (2012) demonstrated that, after excitotoxicity, glucose is directed away from the pentose phosphate pathway toward ATP production via glycolysis. We demonstrate that, while mitochondrial ATP production was not necessary for survival in CGNs at rest, it nevertheless played an important role in ATP recovery after glutamate excitotoxicity. Although $\mathrm{Ca}^{2+}$ sequestration compromises mitochondrial function during excitotoxicity (Nicholls and Budd, 2000), pyruvate supplementation has previously been shown to be protective against excitotoxic injury in CGNs (Vergun et al., 2003; Jekabsons and Nicholls, 2004; but see Khodorov et al., 2012). Our data suggested that mitochondria function sufficiently well to both reduce the initial extent of ATP depletion, and to rapidly and completely restore cytosolic ATP after termination of the excitotoxic stimulus. Indeed, cytosolic $\mathrm{Ca}^{2+}$ influx, leading to increased mitochondrial $\mathrm{Ca}^{2+}$, has been shown to elevate TCA cycle enzymes under physiological conditions, thereby increasing mitochondrial respiration (Hansford and Castro, 1985; McCormack et al., 1990; Gunter et al., 1994). In excitotoxic conditions, increased respiration (Jekabsons and Nicholls, 2004; García et al., 2005) may also elevate mitochondrial ATP production to address the need for energy, emphasizing that, while mitochondria may be impaired during excitotoxicity, they still maintain an active role in ATP recovery. Increased excitotoxic severity may eventually, however, also induce further mitochondrial dysfunction, increasing the likelihood of necrosis (Kushnareva et al., 2005).

AMPK is activated during energetic stress, and restores energetic homeostasis by increasing ATP production pathways while reducing ATP consumption (Hardie et al., 2012). Intriguingly, AMPK activity increased rapidly during excitotoxic injury, but returned to baseline before stimulus termination in some cells, suggesting that AMPK activity was no longer necessary in these cells. AMPK activity has been shown to be both prosurvival and proapoptotic, depending on cell type and the extent of injury, among other factors (McCullough et al., 2005; Weisová et al., 2009; Concannon et al., 2010; Davila et al., 2012). Here, we did not find any correlation between the acute AMPK response measured during glutamate exposure and the subsequent neuronal survival. In addition, we did not observe prolonged AMPK activity in our system after glutamate exposure. It is therefore possible that the intrinsic neuronal response to AMPK activation, rather than the magnitude of the AMPK activation itself, is a determinant of cell fate. However, we need to note that the relative increase in AMPKAR fluorescence ratio during excitotoxicity was low ( $\sim 1.2$-fold), and that we cannot discount the possibility that AMPK activity may remain chronically elevated at levels below the detection limit achievable with this system. In addition, levels of AMPK activity may differ between the cytosol and other intracellular compartments, such as the nucleus (McCullough et al., 2005; Ju et al., 2011; Kodiha et al., 2011; Davila et al., 2012), and this will be investigated in future work.

We also measured single-cell bioenergetics in neurons undergoing OGD, a model of ischemic neuronal injury that has both excitotoxicity-dependent and excitotoxicity-independent components (Goldberg and Choi, 1993). Of note, our single-cell analysis demonstrated that calcium dynamics differed in our OGD treatment paradigm compared with NMDA receptor-mediated excitotoxicity, with a second increase in intracellular calcium measured after restoration of normoxic and normoglycemic conditions. In contrast to excitotoxic injury, ATP levels also did not completely recover after OGD. This is in agreement with population-based ATP measurements in primary neurons after
OGD (Iijima et al., 2003). As mitochondrial ATP production cannot occur in the absence of oxygen, this would largely affect ATP levels in this treatment paradigm. A potential calcium overload after reperfusion may also impact ATP recovery, necessitating additional ATP consumption to restore ionic homeostasis. Subsequent experiments demonstrated that AMPK activity decreased during OGD and only recovered after OGD termination. This decrease in AMPK activity may also affect the ability of the neuron to restore ATP levels. Hence, despite the fact that OGDinduced injury is sensitive to NMDA receptor antagonists (Goldberg and Choi, 1993), the nonexcitotoxic components induced by the hypoxic and hypoglycemic insult impacts on the ability of neurons to recover their bioenergetics.

Using a FRET-based fluorescent reporter, we measured an accumulation of intracellular glucose in single neurons during NMDA receptor-mediated excitotoxicity. Intracellular glucose concentration is a balance between processes that increase glucose and those that decrease glucose. Decreased glucose consumption in combination with steady or increased glucose import could explain the observed net glucose increase in our system. Curiously, however, the removal of extracellular glucose or the inhibition of AMPK with compound C reduced but failed to completely prevent this increase, suggesting additional intracellular glucose sources during excitotoxicity, arising, for example, from glycogen metabolism. While glycogen is normally not present in neurons, they are capable of the synthesis and lysis of glycogen (Vilchez et al., 2007). Interestingly, glycogen metabolism was recently shown to be protective in primary neurons under conditions of hypoxia (Saez et al., 2014). Indeed, the inhibition of glycogen metabolism in our system reduced the time for which the glucose-FRET signal remained elevated, suggesting that the breakdown of glycogen may contribute to the extracellular glucose-independent increase in glucose during excitotoxicity.

Finally, we found that glucose recovery to baseline level was significantly slower than that observed for $\mathrm{Ca}^{2+}$, ATP, and AMPK, suggesting that excess glucose accumulated in the cell during glutamate stimulation. This accumulation may provide the cell with a "fool-proof" mechanism to address latent ATP demand. Perhaps surprisingly, however, we observed that a slower glucose recovery correlated with shorter survival. It is possible that the delayed glucose recovery directly causes earlier death. Accumulated intracellular glucose has been shown to induce aberrant glycogen synthesis in telencephalic neurons, leading to apoptosis (Vilchez et al., 2007). Likewise, slower recovery to baseline may indicate reduced shuttling of glucose into the pentose-phosphate cycle, which ultimately may decrease the antioxidant defense mechanisms during excitotoxic injury (Rodriguez-Rodriguez et al., 2012). Excessive glucose may itself be toxic by increasing reactive oxygen species production, or may lead to increased lactate production and acidosis. Decreased glucose consumption leading to elevated glucose levels may also be an indicator of downstream mitochondrial defects such as $\mathrm{Ca}^{2+}$. induced inhibition of oxidative phosphorylation (Nicholls and Budd, 2000; Kushnareva et al., 2005), although our data indicate that mitochondrial function is retained during the period of glucose elevation. Possibly the most straightforward explanation, however, is that a rapid recovery of glucose homeostasis points to an ability of the neuron to adapt and respond to bioenergetic stressors, and that this plasticity is indicative of underlying neuronal resistance to excitotoxic injury.

In summary, this study is the first to investigate bioenergetic dynamics in single neurons undergoing transient excitotoxicity or oxygen and glucose deprivation. Investigating ATP and glu- 
cose levels along with AMPK activity, our results demonstrated that the bioenergetic recovery of excitotoxic neurons was rapid and highly plastic, and that mitochondrial ATP production participated in this recovery. Our data also indicate that the plasticity of neurons to rapidly adapt to bioenergetic challenges may be a key indicator of neuronal viability.

\section{References}

Ankarcrona M, Dypbukt JM, Bonfoco E, Zhivotovsky B, Orrenius S, Lipton SA, Nicotera P (1995) Glutamate-induced neuronal death: a succession of necrosis or apoptosis depending on mitochondrial function. Neuron 15:961-973. CrossRef Medline

Bonfoco E, Krainc D, Ankarcrona M, Nicotera P, Lipton SA (1995) Apoptosis and necrosis: two distinct events induced, respectively, by mild and intense insults with $\mathrm{N}$-methyl-D-aspartate or nitric oxide/superoxide in cortical cell cultures. Proc Natl Acad Sci U S A 92:7162-7166. CrossRef Medline

Budd SL, Nicholls DG (1996) A reevaluation of the role of mitochondria in neuronal Ca2+ homeostasis. J Neurochem 66:403-411. CrossRef Medline

Castilho RF, Hansson O, Ward MW, Budd SL, Nicholls DG (1998) Mitochondrial control of acute glutamate excitotoxicity in cultured cerebellar granule cells. J Neurosci 18:10277-10286. Medline

Concannon CG, Tuffy LP, Weisová P, Bonner HP, Dávila D, Bonner C, Devocelle MC, Strasser A, Ward MW, Prehn JH (2010) AMP kinasemediated activation of the $\mathrm{BH} 3$-only protein Bim couples energy depletion to stress-induced apoptosis. J Cell Biol 189:83-94. CrossRef Medline

Davila D, Connolly NM, Bonner H, Weisová P, Dussmann H, Concannon CG, Huber HJ, Prehn JH (2012) Two-step activation of FOXO3 by AMPK generates a coherent feed-forward loop determining excitotoxic cell fate. Cell Death Differ 19:1677-1688. CrossRef Medline

D’Orsi B, Bonner H, Tuffy LP, Düssmann H, Woods I, Courtney MJ, Ward MW, Prehn JH (2012) Calpains are downstream effectors of baxdependent excitotoxic apoptosis. J Neurosci 32:1847-1858. CrossRef Medline

Dudek H, Ghosh A, Greenberg ME (2001) Calcium phosphate transfection of DNA into neurons in primary culture. Curr Protoc Neurosci Chapter 3 , Unit 3.11. CrossRef Medline

Fehr M, Lalonde S, Lager I, Wolff MW, Frommer WB (2003) In vivo imaging of the dynamics of glucose uptake in the cytosol of COS-7 cells by fluorescent nanosensors. J Biol Chem 278:19127-19133. CrossRef Medline

García O, Almeida A, Massieu L, Bolaños JP (2005) Increased mitochondrial respiration maintains the mitochondrial membrane potential and promotes survival of cerebellar neurons in an endogenous model of glutamate receptor activation. J Neurochem 92:183-190. CrossRef Medline

Goldberg MP, Choi DW (1993) Combined oxygen and glucose deprivation in cortical cell culture: calcium-dependent and calcium-independent mechanisms of neuronal injury. J Neurosci 13:3510-3524. Medline

Gunter TE, Gunter KK, Sheu SS, Gavin CE (1994) Mitochondrial calcium transport: physiological and pathological relevance. Am J Physiol 267: C313-C339. Medline

Hansford RG, Castro F (1985) Role of Ca2 + in pyruvate dehydrogenase interconversion in brain mitochondria and synaptosomes. Biochem J 227:129-136. Medline

Hardie DG, Ross FA, Hawley SA (2012) AMPK: a nutrient and energy sensor that maintains energy homeostasis. Nat Rev Mol Cell Biol 13:251-262. CrossRef Medline

Hardingham GE, Bading H (2010) Synaptic versus extrasynaptic NMDA receptor signalling: implications for neurodegenerative disorders. Nat Rev Neurosci 11:682-696. CrossRef Medline

Hattori K, Kajimura M, Hishiki T, Nakanishi T, Kubo A, Nagahata Y, Ohmura M, Yachie-Kinoshita A, Matsuura T, Morikawa T, Nakamura T, Setou M, Suematsu M (2010) Paradoxical ATP elevation in ischemic penumbra revealed by quantitative imaging mass spectrometry. Antioxid Redox Signal 13:1157-1167. CrossRef Medline

Iijima T, Mishima T, Tohyama M, Akagawa K, Iwao Y (2003) Mitochondrial membrane potential and intracellular ATP content after transient experimental ischemia in the cultured hippocampal neuron. Neurochem Int 43:263-269. CrossRef Medline

Imamura $\mathrm{H}$, Nhat KP, Togawa H, Saito K, Iino R, Kato-Yamada Y, Nagai T, Noji H (2009) Visualization of ATP levels inside single living cells with fluorescence resonance energy transfer-based genetically encoded indicators. Proc Natl Acad Sci U S A 106:15651-15656. CrossRef Medline

Jekabsons MB, Nicholls DG (2004) In situ respiration and bioenergetic status of mitochondria in primary cerebellar granule neuronal cultures exposed continuously to glutamate. J Biol Chem 279:32989-33000. CrossRef Medline

Ju TC, Chen HM, Lin JT, Chang CP, Chang WC, Kang JJ, Sun CP, Tao MH, Tu PH, Chang C, Dickson DW, Chern Y (2011) Nuclear translocation of AMPK- $\alpha 1$ potentiates striatal neurodegeneration in Huntington's disease. J Cell Biol 194:209-227. CrossRef Medline

Khodorov BI, Mikhailova MM, Bolshakov AP, Surin AM, Sorokina EG, Rozhnev SA, Pinelis VG (2012) Dramatic effect of glycolysis inhibition on the cerebellar granule cells bioenergetics. Biochem (Moscow) Suppl Ser A Membrane Cell Biol 6:186-197. CrossRef

Klingenberg M (2008) The ADP and ATP transport in mitochondria and its carrier. Biochim Biophys Acta 1778:1978-2021. CrossRef Medline

Kodiha M, Ho-Wo-Cheong D, Stochaj U (2011) Pharmacological AMPkinase activators have compartment-specific effects on cell physiology. Am J Physiol Cell Physiol 301:C1307-C1315. CrossRef Medline

Kushnareva YE, Wiley SE, Ward MW, Andreyev AY, Murphy AN (2005) Excitotoxic injury to mitochondria isolated from cultured neurons. J Biol Chem 280:28894-28902. CrossRef Medline

Lankiewicz S, Marc Luetjens C, Truc Bui N, Krohn AJ, Poppe M, Cole GM, Saido TC, Prehn JH (2000) Activation of calpain I converts excitotoxic neuron death into a caspase-independent cell death. J Biol Chem 275: 17064-17071. CrossRef Medline

Lee JM, Zipfel GJ, Choi DW (1999) The changing landscape of ischaemic brain injury mechanisms. Nature 399:A7-A14. CrossRef Medline

Lipton SA, Nicotera P (1998) Calcium, free radicals and excitotoxins in neuronal apoptosis. Cell Calcium 23:165-171. CrossRef Medline

Loewer A, Lahav G (2011) We are all individuals: causes and consequences of non-genetic heterogeneity in mammalian cells. Curr Opin Genet Dev 21:753-758. CrossRef Medline

Marcaida G, Bolaños MD, Grisolía S, Felipo V (1995) Lack of correlation between glutamate-induced depletion of ATP and neuronal death in primary cultures of cerebellum. Brain Res 695:146-150. CrossRef Medline

McCormack JG, Halestrap AP, Denton RM (1990) Role of calcium ions in regulation of mammalian intramitochondrial metabolism. Physiol Rev 70:391-425. Medline

McCullough LD, Zeng Z, Li H, Landree LE, McFadden J, Ronnett GV (2005) Pharmacological inhibition of AMP-activated protein kinase provides neuroprotection in stroke. J Biol Chem 280:20493-20502. CrossRef Medline

Nicholls DG, Budd SL (2000) Mitochondria and neuronal survival. Physiol Rev 80:315-360. Medline

Nicholls DG, Vesce S, Kirk L, Chalmers S (2003) Interactions between mitochondrial bioenergetics and cytoplasmic calcium in cultured cerebellar granule cells. Cell Calcium 34:407-424. CrossRef Medline

Rodriguez-Rodriguez P, Fernandez E, Almeida A, Bolaños JP (2012) Excitotoxic stimulus stabilizes PFKFB3 causing pentose-phosphate pathway to glycolysis switch and neurodegeneration. Cell Death Differ 19:15821589. CrossRef Medline

Rodriguez-Rodriguez P, Almeida A, Bolaños JP (2013) Brain energy metabolism in glutamate-receptor activation and excitotoxicity: role for APC/ $\mathrm{C}$-Cdh1 in the balance glycolysis/pentose phosphate pathway. Neurochem Int 62:750-756. CrossRef Medline

Saez I, Duran J, Sinadinos C, Beltran A, Yanes O, Tevy MF, Martinez-Pons C, Milan M, Guinovart JJ (2014) Neurons have an active glycogen metabolism that contributes to tolerance to hypoxia. J Cereb Blood Flow Metab 34:945-955. CrossRef Medline

Takanaga H, Chaudhuri B, Frommer WB (2008) GLUT1 and GLUT9 as major contributors to glucose influx in HepG2 cells identified by a high sensitivity intramolecular FRET glucose sensor. Biochim Biophys Acta 1778:1091-1099. CrossRef Medline

Takemoto K, Nagai T, Miyawaki A, Miura M (2003) Spatio-temporal activation of caspase revealed by indicator that is insensitive to environmental effects. J Cell Biol 160:235-243. CrossRef Medline

Tsou P, Zheng B, Hsu CH, Sasaki AT, Cantley LC (2011) A fluorescent reporter of AMPK activity and cellular energy stress. Cell Metab 13:476486. CrossRef Medline

Vergun O, Han YY, Reynolds IJ (2003) Glucose deprivation produces a pro- 
longed increase in sensitivity to glutamate in cultured rat cortical neurons. Exp Neurol 183:682-694. CrossRef Medline

Vilchez D, Ros S, Cifuentes D, Pujadas L, Vallès J, García-Fojeda B, Criado-García O, Fernández-Sánchez E, Medraño-Fernández I, Domínguez J, García-Rocha M, Soriano E, Rodríguez de Córdoba S, Guinovart JJ (2007) Mechanism suppressing glycogen synthesis in neurons and its demise in progressive myoclonus epilepsy. Nat Neurosci 10:1407-1413. CrossRef Medline

Wang Y, Qin ZH (2010) Molecular and cellular mechanisms of excitotoxic neuronal death. Apoptosis 15:1382-1402. CrossRef Medline

Ward MW, Rego AC, Frenguelli BG, Nicholls DG (2000) Mitochondrial membrane potential and glutamate excitotoxicity in cultured cerebellar granule cells. J Neurosci 20:7208-7219. Medline
Ward MW, Rehm M, Duessmann H, Kacmar S, Concannon CG, Prehn JH (2006) Real time single cell analysis of Bid cleavage and Bid translocation during caspase-dependent and neuronal caspase-independent apoptosis. J Biol Chem 281:5837-5844. Medline

Ward MW, Huber HJ, Weisová P, Düssmann H, Nicholls DG, Prehn JH (2007) Mitochondrial and plasma membrane potential of cultured cerebellar neurons during glutamate-induced necrosis, apoptosis, and tolerance. J Neurosci 27:8238-8249. CrossRef Medline

Weisová P, Concannon CG, DeVocelle M, Prehn JH, Ward MW (2009) Regulation of glucose transporter 3 surface expression by the AMPactivated protein kinase mediates tolerance to glutamate excitation in neurons. J Neurosci 29:2997-3008. CrossRef Medline 